\title{
CSHP Professional Practice Conference 2018: Poster Abstracts / Conférence sur la pratique professionnelle 2018 de la SCPH : résumés des affiches
}

Facilitated Poster Sessions: Discussions of original research and pharmacy practice projects

Séance animée de présentations par affiches : Discussions sur des projets de recherche originale et des projets dans le domaine de la pratique pharmaceutique

\section{Sunday, February 4, $2018 \bullet$ Dimanche 4 février 2018}

Infectious Diseases / Antimicrobial Stewardship

1. Falsely Elevated Vancomycin Concentrations in a Patient Not Receiving Vancomycin

2. A Case of Vancomycin-Induced Thrombocytopenia

3. Successful Treatment of Chronic Spinal Osteomyelitis Caused by Multidrug Resistant Pseudomonas aeruginosa with Ceftolozane-Tazobactam and Surgical Intervention

4. Clinical Burden of Antibiotic Resistance Following Implementation of a Multidisciplinary Antimicrobial Stewardship Initiative in a Major Tertiary Care Center: A Controlled Interrupted Time Series Analysis Over 14 Years

5. Antibiotic Utilization Feedback Reports on General Medicine: A Qualitative Assessment

Pharmacy Practice / Patient-Centred Care / Pharmacy Education \#1

1. A Descriptive Analysis of an Alternate-Level-of-Care Patient Cohort: Comprehensive Medication Overview

2. Pharmacist Actual and Perceived Priority Interventions in the Emergency Department: An Observational Study and Survey Questionnaire

3. Patient Preferences for Atrial Fibrillation Stroke Prevention Therapy Using an Individualized Risks and Preferences-Based Decision Aid

4. Optimizing Patient Education of Oncology Medications: A Patient Perspective (award—see page 55)

5. Informing Patients and Families about Storage and Disposal of Opioids

Medication Utilization, Effectiveness, and Safety

1. Pan-Canadian Trends in the Prescribing of Opioids, 2012 to 2016

2. Cardiac Arrest after Acute on Chronic Colchicine Toxicity

3. Mandatory Quality Related Events Reporting in Canada: A Province-wide Review over 7 Years

4. Analysis of Smart Pump Continuous Quality Improvement Data across Multiple Organizations

5. Comparison of Weight-Based versus Fixed-Dose Norepinephrine Dosing in Patients with Septic Shock

Drug Stability, Drug Shortages, Pharmacokinetics, and Occupational Exposure

1. Varying Ammonia Levels with Two Formulations of Sodium Phenylbutyrate

2. Stability of $8.4 \%$ Injectable Sodium Bicarbonate When Stored at Room Temperature

3. Stability of Ropivacaine $2.7 \mathrm{mg} / \mathrm{mL}$, Morphine $0.091 \mathrm{mg} / \mathrm{mL}$ and Ketorolac $0.27 \mathrm{mg} / \mathrm{mL}$ in Polyvinyl Chloride Bags at $4^{\circ} \mathrm{C}$

4. Étude descriptive de la contamination urinaire de travailleurs exposés au cyclophosphamide, à l'ifosfamide, au méthotrexate et au fluorouracile

5. Stability of 10, 40 and $200 \mathrm{mcg} / \mathrm{mL}$ Hydromorphone Solutions Stored in Syringes at Room Temperature $\left(23^{\circ} \mathrm{C}\right)$

Pharmacy Practice / Patient-Centred Care / Pharmacy Education \#2

1. Atrial Fibrillation Patient Knowledge Gaps: A Systematic Review

2. A Feasibility Study: Implementing a Medication Adherence Contract in Kidney Transplant Recipients Followed by an Outpatient Transplant Clinic

3. An Emerging Challenge: Pharmacists' Ability to Counsel a Growing Immigrant Population

4. Toxicology Education Needs of Emergency Department Pharmacists

5. Implementation of an Overnight Pharmacist Mode
Medication Utilization, Effectiveness, and Safety / Drug Stability and Shortages

1. A Literature-Based Algorithm for the Assessment, Management and Monitoring of Drug-Induced QTc Prolongation in the Psychiatric Population

2. Provincial Smart Pump Program Improves Patient Safety

3. A Multi-Incident Analysis on Medication Incidents Associated with Patient Harm

4. Insourcing High-Risk Sterile Compounded Injectables: Development of In-House Capacity to Produce High-Quality Injectables during the Sodium Bicarbonate Shortage

5. Coordinating a Response to a Critical Drug Shortage: Experience with Sodium Bicarbonate

\section{Monday, February 5, 2018 - Lundi 5 février 2018}

Infectious Diseases / Antimicrobial Stewardship

1. Development and Implementation of a Mobile Antimicrobial Handbook App at a Tertiary Teaching Hospital

2. Retrospective Multicentre Cohort Study Comparing Safety and Efficacy Outcomes with Intermittent Infusion versus Continuous Infusion Vancomycin

3. Evaluation of Dosing Strategies with Meropenem using Monte Carlo Simulations against Bacteria with Raised MIC

4. Development of an HIV National Clinical Observership Program for Pharmacists

5. An Innovative In-House Developed Access ${ }^{\circledR}$ Database to Capture and Analyze Antimicrobial Stewardship Interventions

Pharmacy Practice / Patient-Centred Care / Pharmacy Education

1. Performance of an Innovative Patient Decision Aid for Atrial Fibrillation Stroke Prevention Therapy

2. Novel Student-Preceptor Models in Pharmacy Education: A Qualitative Analysis of the PharmD Student Experience

3. Evaluation of Physical Assessment Education for Practicing Pharmacists: A Cross-Sectional Study

4. International Normalized Ratio Point of Care Testing in an Outpatient Anticoagulation Clinic and the Impact on the Patient Experience: A Quality Improvement Study

Medication Utilization, Effectiveness, and Safety \#1

1. Case Report: Fatal Arrhythmia in a Patient Receiving an Aconite Herbal Product

2. A Survey of the Use of Cannabis in Children at a Tertiary Teaching Hospital

3. Current Identification and Management Practices of Steroid-Induced Hyperglycemia in Brain and Spinal Tumour Patients on a Neurosurgical Unit

4. Clonidine for Sedation in Critically Ill Adults: A Retrospective Chart Review

5. The Effect of Preoperative Cannabis Use on Opioid Consumption Following Surgery: A Cohort Analysis

Drug Stability, Drug Shortages, Pharmacokinetics, and Occupational Exposure

1. Stability of Extemporaneously-Compounded Temozolomide $10 \mathrm{mg} / \mathrm{mL}$ Suspension in Oral Mix SF in Glass and Plastic Bottles and Plastic Syringes

2. Stability of 20,40 and $50 \mathrm{mcg} / \mathrm{mL}$ Fentanyl Solutions Stored in Syringes at Room Temperature $\left(23^{\circ} \mathrm{C}\right)$

3. Stability of Generic Formulations of Bortezomib 1.0 and $2.5 \mathrm{mg} / \mathrm{mL}$ in Vials and Syringes Stored $4^{\circ} \mathrm{C}$ and Room Temperature $\left(23^{\circ} \mathrm{C}\right)$

4. Stability of 4 and $10 \mathrm{mcg} / \mathrm{mL}$ Remifentanil Solutions Stored in Syringes at Room Temperature $\left(23^{\circ} \mathrm{C}\right)$

5. Satisfaction des établissements de santé suite à la mise en place d'une plateforme web de surveillance environnementale 


\section{PPC 2018 POSTER ABSTRACTS / RÉSUMÉS DES AFFICHES DE LA CPP 2018}

Medication Utilization, Effectiveness, and Safety \#2

1. Medication Fluids in the Intensive Care Unit

2. Drug Use Evaluation of Oxycodone at a Canadian Teaching Hospital

3. Inpatient Insulin Stewardship Program: A Baseline Needs Assessment

4. Long-Term Beta-Blockers after Myocardial Infarction in the Contemporary Era: A Systematic Review

5. Clinical Guide for Pharmacists to Evaluate Risks and Manage QTc Prolongation Drug-Drug Interactions

Infectious Diseases / Antimicrobial Stewardship/ Pharmacy Practice

1. Trickle-Down Antimicrobial Stewardship: Reduction in Long-Term Care Resistance Rates Following Implementation of a Prospective Audit and Feedback Intervention in the Adjacent Acute Care Hospital

2. Enhancing Mental Health Services through Hospital Outpatient Pharmacy and Assertive Community Treatment Team Collaboration

3. Vancomycin Every 4 hours in Paediatric Patients: A Case Series

4. Comparison of Clinical Pharmacy Services in General Medicine and Surgery Patients: A Workload Measurement Study

\section{Tuesday, February 6, $2018 \bullet$ Mardi 6 février 2018}

Infectious Diseases / Antimicrobial Stewardship

1. Validation of a Screening Tool to Assist in the Early Identification of Bloodstream Infection in Older Patients

2. Patterns of Antimicrobial Use in an Outpatient Hemodialysis Unit

3. Impact of an Antimicrobial Stewardship Bloodstream Infection Surveillance Program in Hospitalized Patients

4. Epidemiology of Carbapenemase-Producing Enterobacteriaceae Bacteremia and Evaluation of Antimicrobial Prescribing Practices in a Community . Hospital Setting

5. Development and Implementation of a Provincial Beta-Lactam Allergy Management Initiative

Pharmacy Practice / Patient-Centred Care/ Pharmacy Education \#1

1. Factors Affecting Time to Fill Antiplatelet Therapy for Patients Discharged from Hospital

2. Evaluation of the Impact of Pharmacist-Led Penicillin Allergy Assessments on Antibiotic Utilization in a Large Community Teaching Hospital

3. Pharmacy Technician Continuing Education Program at a Large Teaching Hospital

4. 5 Questions to Ask about Your Medications

5. A Pharmacy-Led Interdisciplinary Teaching Model in Specialized Pharmacotherapy: An HIV Pharmacy Rotation for Medical Residents
Medication Utilization, Effectiveness, and Safety / Drug Stability

1. Influence of Manufacturer on Cefazolin Stability

2. Opioid Selection in the Neonatal Intensive Care Unit: Morphine versus Fentanyl: Impact on Total Opioid Exposure and Time to Enteral Feeds

3. The Prevalence of Mortality due to Rebound Toxicity after "Treat and Release” Practices In Prehospital Opiate Overdose Care: A Systematic Review

4. A Pan-Canadian Study on the Compounded Medicines Most in Need of Commercialized Oral Pediatric Formulations

5. A Comparison of Intravenous Iron Dosing Regimens for Anemia Management in Patients Undergoing Hemodialysis

Pharmacy Practice / Patient-Centred Care / Pharmacy Education \#2

1. User Satisfaction Regarding Standard Assessment Tool for Field-Based Pharmacy Training

2. Pharmacy Student-Led Best Possible Medication History Quality Audit

3. A Work-Sampling Study of Clinical Pharmacists

4. Development and Evaluation of an Anticoagulation Education Program for Pharmacists

5. Évaluation de l'oculométrie comme outil de rétroaction en validation pharmaceutique : étude pilote

Pharmacy Practice / Patient-Centred Care / Pharmacy Education \#3

1. Qualitative Thematic Analysis of Interprofessional Perspectives on Clinical Pharmacy Key Performance Indicators

2. Enabling Expanded Scope in Hospital Practice: Implementation of a Pharmacist Modification Orders Protocol

3. Design, Implementation, and Evaluation of a Clinical Pharmacy Key Performance Indicator Tracker: DIE-cpKPI Study

4. Assessing the Perception and Implementation of Continuous Quality Improvement in Pharmacy Professionals: A Pre-Safety IQ Initiative

5. From "Dot" to "Dot Com": Navigating Pharmacist Handoff in a Digital Era 


\section{Falsely Elevated Vancomycin Concentrations in a Patient Not Receiving Vancomycin}

Tsoi VK ${ }^{1}$, Bhayana $V^{1,2}$, Bombassaro AM $M^{1,2}$, Tirona $R^{1,2}$, Betchen $D^{1}$, Kittanakom $S^{3,4}$

${ }^{1}$ London Health Sciences Centre, London, ON

${ }^{2}$ Western University, London, ON

${ }^{3}$ William Osler Health System, Brampton, ON

${ }^{4}$ University of Toronto, Toronto, $O N$

Background: Therapeutic drug monitoring (TDM) of vancomycin is recommended by the Infectious Diseases Society of America for efficacy and toxicity. Immunoassays are commonly used in diagnostic laboratories to determine serum vancomycin concentrations. A case of falsely elevated vancomycin serum concentrations, in a patient not receiving vancomycin therapy, is described.

Case Description: An elderly female was prescribed vancomycin and imipenem for a suspected septic knee. A blood sample was inadvertently collected prior to her receiving vancomycin, and the concentration reported as $36.10 \mathrm{mg} / \mathrm{L}$ (Roche Modular P analyzer). Repeated serum sampling over the subsequent 48 hours, despite not receiving vancomycin, yielded similar concentrations $(33.10-35.30 \mathrm{mg} / \mathrm{L})$. Eight months later, she was again found to have an elevated vancomycin concentration $(32.9 \mathrm{mg} / \mathrm{L})$ in the absence of therapy.

Assessment of Causality: The patient sample was tested for vancomycin using liquid chromatography-tandem mass spectrometry (LC-MS/MS) as the gold-standard, and by four alternate commercial immunoassay platforms. Vancomycin was undetectable by LC-MS/MS. The immunoassays showed undetectable to high $(35 \mathrm{mg} / \mathrm{L})$ concentrations. The falsely elevated concentration observed by our Roche method was investigated for interference by concurrent medications and endogenous substances, such as paraproteins and human anti-mouse antibodies. Polyethylene glycol precipitation and heat inactivation resulted in the removal of the interference responsible for the falsely elevated concentration.

Literature Review: A single case of a spuriously high vancomycin concentration before receiving the drug, secondary to suspected endogenous interference, has been reported. However, to our knowledge, this is the first report of falsely elevated vancomycin concentrations on the Roche Modular $\mathrm{P}$ analyzer in a patient not receiving vancomycin.

Importance to Practitioners: Vancomycin immunoassays are robust in facilitating TDM, but are susceptible to cross-reactivity. Spurious concentrations may lead to unnecessary dose adjustments, impacting efficacy or toxicity. Assay interference should be considered and laboratory professionals contacted when vancomycin levels do not correlate with clinical expectations.

\section{A Case of Vancomycin-Induced Thrombocytopenia}

Szabolcs $N^{1}$, Neilans $L^{2}$, Bombassaro $A M^{1,3}$, Xenocostas $A^{1,3}$, Kinney $J^{1}$

${ }^{1}$ London Health Sciences Centre, London, ON

${ }^{2}$ Parkwood Institute, St. Joseph's Health Care, London, ON

${ }^{3}$ Western University, London, ON

Background: Vancomycin has been implicated in causing cytopenias. While neutropenia is well described, vancomycin-induced thrombocytopenia is rare and may be overlooked in an initial thrombocytopenia workup, increasing the risk of potentially life-threatening bleeding.

Case Description: A 41 year-old female underwent revision of a previous spinal decompression surgery. Irrigation and debridement 15 days later found complications of dural leaks, pseudomeningocele and necrotic bone. She was empirically started on vancomycin and meropenem. Her baseline platelet count was 349 (reference range: 150-400x109/L).
Between days 4 and 9 from vancomycin initiation her platelets decreased from 300 to 62 , reaching a nadir of 15 on day 12. Antibiotics were discontinued. The platelets rebounded to 68 the following day and meropenem was resumed for a total of 6 weeks. The patient was transferred to another institution where vancomycin was restarted on day 19 with a platelet count of 372 , which decreased to 78 by the following morning. Vancomycin was discontinued and the platelets rebounded to 120 by day 23 .

Assessment of Causality: The Naranjo scale score of 10 identified vancomycin as a definite cause of the thrombocytopenia. The reaction resolved with drug withdrawal and recurred within 24 hours of rechallenge. Flow cytometry testing for drug-induced thrombocytopenia was positive with vancomycin-dependent immunoglobulin $G$ binding to platelets detected in the post-exposure but not in the pre-exposure serum.

Literature Review: A retrospective review published in 2017 identified 30 case reports of vancomycin-induced thrombocytopenia. A study investigating a case series of patients receiving vancomycin detected vancomycin-dependent antiplatelet antibodies only in those in whom thrombocytopenia developed.

Importance to Practitioners: Although vancomycin-induced thrombocytopenia is a rare side effect, prompt recognition and drug discontinuation may minimize the risk of a life-threatening bleed. At least weekly monitoring of platelets has been recommended and patient counseling regarding risks of future exposure is important.

\section{Successful Treatment of Chronic Spinal Osteomyelitis Caused by Multidrug Resistant Pseudomonas aeruginosa with Ceftolozane-Tazobactam and Surgical Intervention}

Hooper $C^{1}$, Elsayed $S^{1,2}$, Bombassaro AM $M^{1,2}$, Bailey $C^{1,2}$, Bondy $L^{1,2}$

${ }^{1}$ London Health Sciences Centre, London, ON

${ }^{2}$ Western University, London, ON

Background: Ceftolozane-tazobactam is a novel intravenous antibiotic combining an anti-pseudomonal cephalosporin with a beta-lactamase inhibitor. It is indicated for complicated intra-abdominal and urinary tract infections. Information on the use of ceftolozane-tazobactam in osteomyelitis is limited and is the focus of this case report.

Case Description: A 67 year-old morbidly obese female with chronic draining wounds, refractory to multiple courses of antibiotics after spinal deformity correction three years prior, was admitted for surgical irrigation and debridement. Existing rods and hardware were removed and pockets of purulent material were found throughout the spinal exposure. Intraoperative cultures grew Streptococcus anginosus and multidrug resistant (MDR) Pseudomonas aeruginosa sensitive only to aminoglycosides. A clinical diagnosis of chronic osteomyelitis was made. Treatment with tobramycin and meropenem was initiated. Additional testing by disk diffusion demonstrated susceptibility to ceftolozane-tazobactam. Treatment with a 6 week course of ceftolozane-tazobactam $3 \mathrm{~g}$ intravenously every 8 hours was well tolerated.

Assessment of Causality: At days 4 and 31 after the start of ceftolozanetazobactam, intraoperative cultures were negative for the previously identified organisms. At last follow up, 6 months after the initial surgery, the patient's wounds had healed completely. She was clinically well and not receiving antibiotic therapy.

Literature Review: The 5 published case reports using ceftolozanetazobactam for osteomyelitis have involvedMDR Pseudomonas (4) and MDR Stenotrophomonas (1). Doses varied from $750 \mathrm{mg}$ (adjusted for renal impairment) to $3 \mathrm{~g}$ intravenously every 8 hours for at least 6 weeks. Three 
cases reported clinical success at 3 to 18 months of follow-up while two reports did not include outcome information.

Importance to Practitioners: MDR organisms remain challenging to treat, particularly when involving deep seated infections such as osteomyelitis. Sharing this experience of using ceftolozane-tazobactam combined with surgical intervention is a valuable addition to the existing literature for clinicians faced with similar clinical scenarios.

\section{Clinical Burden of Antibiotic Resistance Following Implementation of a Multidisciplinary Antimicrobial Stewardship Initiative in a Major Tertiary Care Center: A Controlled Interrupted Time Series Analysis Over 14 Years}

Peragine $C^{1,2}$, Walker $S A N^{1,2,3,4^{*}}$, Leis $J A^{3,4,5,6,7}$, Simor $A E^{3,4,5,6}$

${ }^{1}$ Department of Pharmacy, Sunnybrook Health Sciences Centre, Toronto, ON

${ }^{2}$ Leslie L. Dan Faculty of Pharmacy, University of Toronto, Toronto, ON

${ }^{3}$ Division of Infectious Diseases, Sunnybrook Health Sciences Centre,

Toronto, $O N$

${ }^{4}$ Sunnybrook Health Sciences Centre Research Institute, Sunnybrook Health Sciences Centre, Toronto, ON

${ }^{5}$ Faculty of Medicine, University of Toronto, Toronto, $O N$

${ }^{6}$ Division of Infectious Diseases, Department of Medicine, Toronto, ON

${ }^{7}$ Department of Infection Prevention and Control, Toronto, ON

* Senior Author; sequence determines credit approach to authorship

Background: Our institution launched a multidisciplinary prospective audit-and-feedback (PAF) Antimicrobial Stewardship program (ASP) in October 2009. Although reducing antimicrobial resistance (AMR) is a major incentive for ASPs, a paucity of high quality data evaluating the impact of ASPs on microbial outcomes remains.

Objective: The objective was to evaluate changes in institutional AMR and antimicrobial use (AMU) in the 7 years following PAF-ASP implementation.

Methods: Patient-level microbiologic data (clinical isolates of aerobic gram-negative bacteria, Staphylococcus aureus, and Enterococcus spp.) and antibiotic-use data were obtained over the 14 year study period. Program impact was assessed by evaluating changes in institutional AMR and AMU trends ( $\Delta$ slope) between the pre- (October 2002 - September 2009) and post-intervention (October 2009 - September 2016) periods using interrupted time-series analyses with segmented regression. Incidence of hospital-acquired isolates resistant to at least one therapeutically active antibiotic agent per month standardized to 10,000 patient days (PD) was assessed as the primary outcome. Change in the incidence of antibiotic-resistant community-acquired isolates was evaluated as an AMR control. Changes in inpatient AMU were quantified using antibiotic days of therapy (DOT) (all patients, all antibiotic agents)/ 10,000 PD per month.

Results: A significant reduction in nosocomial AMR $(\Delta$ slope $=-0.7$ resistant hospital-acquired isolates/10,000 $\mathrm{PD} /$ month, $\mathrm{p}<0.001)$ and inpatient $\mathrm{AMU}(\Delta$ slope $=-36$ antibiotic $\mathrm{DOT} / 10,000 \mathrm{PD} / \mathrm{month}$, $\mathrm{p}<0.001)$ was found. Conversely, community-acquired AMR increased significantly over the same period $(\Delta$ slope $=+0.2$ resistant communityacquired isolates $/ 10,000 \mathrm{PD} /$ month, $\mathrm{p}=0.03)$.

Conclusions: Time series modelling revealed that implementation of a PAF-ASP was associated with a significant reduction in nosocomial AMR and inpatient AMU. The identified increase in community acquired AMR further strengthens the causal inference of our findings, since an institutional ASP would not be expected to impact community AMR and the observed significant rise in community AMR is in keeping with the known global crisis of rising AMR in the community.

\section{Antibiotic Utilization Feedback Reports on General Medicine: A Qualitative Assessment}

Rai $S^{1,2}$, Elligsen $M^{1}$, Lo ${ }^{1}$, Walker $S A N^{1,2,3,4}$, Peragine $C^{1,2}$, Alattas $N^{5}$, Daneman $N^{4,5}$, Leis $J A^{4,5}$

${ }^{1}$ Department of Pharmacy, Sunnybrook Health Sciences Centre, Toronto, ON

${ }^{2}$ Leslie Dan Faculty of Pharmacy, University of Toronto, Toronto, $O N$

${ }^{3}$ Division of Infectious Diseases, Sunnybrook Health Sciences Centre, Toronto, ON

${ }^{4}$ Sunnybrook Health Sciences Centre Research Institute, Sunnybrook Health Sciences Centre, Toronto, ON

${ }^{5}$ Faculty of Medicine, University of Toronto, Toronto, $O N$

Background: Between 30 to $50 \%$ of antibiotic prescriptions are potentially unnecessary and these inappropriate prescriptions drive antimicrobial resistance. Evidence suggests that despite treating similar patient populations, antibiotic prescribing practices can vary dramatically among prescribers. Peer comparison is an increasingly recognized behavioural intervention to improve physician prescribing practices.

Objectives: We sought to measure prescriber-level antibiotic utilization among general internal medicine (GIM) attending physicians to provide peer comparison reports, and assess any impact on physician practices.

Methods: Antibiotic Days of Therapy (DOT) per 1000 patient days under the care of each GIM physician was calculated using our antimicrobial stewardship database. We included all attending physicians with at least 3 weeks of inpatient service during the study period (January 2nd 2015 to June 27th 2016). Readmission or death within 30 days for patients who received at least one antibiotic was used as a balancing measure. Anonymous, personalized feedback reports were emailed to GIM physicians describing their antibiotic prescribing practices compared to their peers. An electronic survey was conducted 2 months later to assess perceptions regarding feedback and any contemplated changes in practice as a result of the report.

Results: Among 17 GIM physicians, DOT per 1000 patient days varied from 316 to 569 . Despite these differences, there was no difference in 30 day readmission or death among patients receiving antibiotics. Of the 10 physicians that completed the feedback survey (response rate 63\%), 9 prescribers $(90 \%)$ found the report represented their practice while $4(40 \%)$ contemplated changes in practice.

Conclusions: Provider-level antibiotic utilization at our institution confirmed wide variation in prescribing practices without an obvious difference in patient outcomes. The majority of physicians surveyed felt that antibiotic prescribing feedback reports were useful, but longer term follow up is needed to evaluate impact on antibiotic prescribing practices.

\section{A Descriptive Analysis of an Alternate-Level-of-Care Patient Cohort: Comprehensive Medication Overview}

Azimi $M^{1,2}$, Burry L L,2,3, Duclos $C^{1,2}$, Pelc J J Upshur R ${ }^{4,5,6}$

${ }^{1}$ Leslie Dan Faculty of Pharmacy, University of Toronto, Toronto, ON

${ }^{2}$ Department of Pharmacy, Mount Sinai Hospital, Toronto, ON

${ }^{3}$ Division of Intensive Care Unit, Mount Sinai Hospital, Toronto, ON

${ }^{4}$ Department of Medicine, Bridgepoint Active Healthcare, Toronto, ON

${ }^{5}$ Division of Clinical Public Health, Dalla Lana School of Public Health,

Toronto, $O N$

${ }^{6}$ Department of Family and Community Medicine, Toronto, ON

Background: Alternate Level of Care (ALC) patients are hospitalized, not receiving active medical care waiting to be transferred to another facility. Many older patients are on complex, often inappropriate 
medications. To our knowledge, there has been no analysis of ALC medication regimens. We conducted an audit of all ALC patients within a tertiary institution.

Objectives: (1) to analyze health-related parameters and obtain a descriptive representation of ALC patients currently admitted at our institution; (2) to cross-reference medications to 3 existing literature references for potentially inappropriate medications (PIMs) (Beer's list, STOPP/ START and ISMP high alert medications); and (3) to assess the appropriateness of medication regimens.

Methods: We conducted a cross-sectional audit of ALC patients in June 2017 as a phase I quality improvement project. Data was collected with a standardized case report form to obtain a descriptive representation of ALC patients. Medications were categorized and coded based on the 3 PIM lists. Data was analyzed by calculating mean, median and IQR where appropriate.

Results: Eighty-two ALC patients were included in this study. The mean age of patients was $75.6( \pm 15)$ years. Female patients accounted for $52.4 \%$ of our population. Patients had $6.4(\mathrm{SD} \pm 3)$ chronic conditions, and were prescribed $12.8(\mathrm{SD} \pm 7)$ medications; $28.9 \%$ of patients were prescribed $\geq 1$ drug from 7 different classes of medications concurrently. PIMs were most frequent on the Beer's list with an average of $3.9(\mathrm{SD} \pm 3)$ medications/patient. One patient had zero medications flagged as PIM from any of the 3 PIM lists.

Conclusions: ALC patients at our institution have 6 chronic conditions managed with at least 12 medications, many of which are PIMs. This data will inform next steps to make recommendations to simplify, reduce or discontinue medications in which there is an unclear indication, lack of effectiveness or evidence of potential harm.

\section{Pharmacist Actual and Perceived Priority Interventions in the Emergency Department: An Observational Study and Survey Questionnaire}

Dalen $D^{1,2}$, Kelly $J^{1}$, Zed $P^{2,3}$, Gorman $S^{1,2}$, Nevers $W^{1}$, Slavik ${ }^{1,2}$, Harris $D^{1,3}$

${ }^{1}$ Pharmacy Services, Interior Health Authority, Kelowna, BC

${ }^{2}$ Faculty of Pharmaceutical Sciences, University of British Columbia,

Vancouver, $B C$

${ }^{3}$ Department of Emergency Medicine, University of British Columbia, Vancouver, $B C$

Background: Emergency Department (ED) pharmacists are a finite resource and activities with higher value should be prioritized. Literature shows that clinical pharmacists who resolve drug therapy problems, called pharmacist interventions (PI), improve patient outcomes and reduce health-resource utilization. Tracking high quality PI and surveying pharmacists to understand their perceptions of their practice will help us establish baseline ED pharmacist practice behaviour and identify potential gaps in ED pharmacist services delivery.

Objectives: To describe the number of PI completed by ED pharmacists in a Canadian health authority, to describe the proportion of interventions that were priority disease PI (PD-PI) and quality indicator PI (QI-PI), and to identify ED pharmacist perceptions of which patients they felt they should be providing prioritized care to and which activities they felt they should be completing.

Methods: This study consisted of two parts; a retrospective PI tracking review and an internet-based survey. PI tracking data was captured from three EDs with dedicated clinical pharmacist coverage over a one year period. An electronic survey was distributed to a convenience sample of Canadian ED pharmacists. Data was analyzed using descriptive statistics.
Results: A total of 2043 PI were completed. Of these, 1127 (54.4\%) were PD-PI and 473 (22.9\%) were QI-PI. Survey participants agreed that many priority diseases and high-value activities (QI-PI and clinical pharmacist key performance indicators [cpKPI]) previously established in a general medicine population should be prioritized in the ED, but not all. Several potential new ED-specific priority diseases were identified.

Conclusions: ED pharmacists in a Canadian health authority are performing impactful, high quality interventions for patients with priority diseases. Surveyed pharmacists identified gaps in current definitions of priority diseases and high value pharmacist activities specific to the ED. Our findings support the presence of unique opportunities for ED clinical pharmacists to provide prioritized, impactful care.

\section{Patient Preferences for Atrial Fibrillation Stroke Prevention Therapy Using an Individualized Risks and Preferences-Based Decision Aid}

Loewen $P^{l}$, Bansback $N^{1}$, Andrade ${ }^{1,2}$, Bonet $B^{4}$, Deyell $M^{1,3}$, Hicklin $I^{1}$, Kapanen $A^{1}$, Kwan $L^{4}$, Lynd $L^{1}$, McLean $A^{1}, M c$ Gillivray ${ }^{2}$, Salmasi $S^{1}$

${ }^{1}$ The University of British Columbia, Vancouver, $B C$

${ }^{2}$ Vancouver Coastal Health, Vancouver, BC

${ }^{3}$ Providence Health Care, Vancouver, BC

${ }^{4}$ Fraser Health, Vancouver, BC

Background: Many antithrombotic therapies are effective for atrial fibrillation (AF) stroke prevention, but choosing is complex because patients must make trade-offs based on their values and preferences. To address this, we have developed a decision aid (DA) that integrates AF education, individualized stroke and bleeding risk estimates, clinical trial evidence, a best-worst scaling discrete choice experiment (BWS-DCE), and quantification of the alignment between therapy options and patient preferences.

Objective: To assess the preferences of AF patients and their consequent stroke prevention therapy choices.

Methods: Prospective observational study of patients with AF or at risk of AF (age >50 y/o). Participants completed a pre-DA questionnaire, the $\mathrm{DA}$, and a post-DA questionnaire. The DA integrated each participant's stroke and major bleed risk with the results of a 9-attribute BWS-DCE to generate individualized preference scores for each therapy option. Participants made therapy choices based on the results presented.

Results: 38 participants (mean age 71 ) completed the study. $61 \%$ had CHA2DS2-VASc score $>1$ and 32\% had HAS-BLED score $>2.70 \%$ were on OAC. Stroke and intracranial hemorrhage were the highest and daily doses and cost were the lowest-weighted attributes. 54\% of participants changed their preferred treatment after using the DA. $68 \%$ and $12 \%$ chose the highest-scored therapy option on decision 1 (no therapy, ASA, or OAC) and decision 2 (choice of OAC), respectively. In $54 \%$, the direction of therapy change was to de-intensify (OAC to ASA or ASA to no therapy). $47 \%$ of OAC users preferred a different OAC than current.

Conclusions: Using a DA that included individualized risk estimates, values clarification, and scoring of therapy options, most AF patients decided they preferred a different therapy than the one currently prescribed. The DA requires refinement for choice of OAC. Using the DA could be a valuable facilitator of shared decision-making in AF patients. 


\section{Informing Patients and Families about Storage and Disposal of Opioids}

Hyland $B^{1}$, Fan $M^{1}$, Hamilton $M^{2}$, Reding $R^{1}$, Trbovich $P^{1,3}$

${ }^{1}$ Research and Innovation, North York General Hospital, Toronto, ON

${ }^{2}$ Institute for Safe Medication Practices Canada, Toronto, ON

${ }^{3}$ Institute of Health Policy, Management and Evaluation, University

of Toronto, Toronto, ON

Background: Unsafe storage or disposal of medications including opioids has resulted in accidental poisonings, inappropriate use, and theft in patient's homes. Increasing demand for home-based end-of-life care underscores the opportunity to provide guidance to patients and families.

Objectives: The project objectives were to 1) identify preferred practices for safe home-based opioid storage and disposal, and 2) develop and refine educational materials to support patients, families, and healthcare providers.

Methods: Preferred practices regarding home-based opioid storage and disposal were identified via thematic analysis of 155 out of 1285 articles screened for inclusion and 20 multi-disciplinary stakeholder interviews. An information card (English version below) was developed using usability testing with research ethics board approval.

Results: Thematic analysis revealed that patient/family education was not consistently provided by the healthcare system, nor reliably supported with educational tools.

Complementary preferred practices were identified:

1. Homecare service providers pick up unused medications

2. Pharmacists providing in-home medication reviews pick up unused medications

3. Patients/families return medications to a pharmacy; they can contact the XXX to identify Canadian pharmacies participating in 'returns programs'

Given variability in clinical practices, an information card was developed to support preferred practice \#3 above. Usability testing revealed opportunities for improvement; the information card was refined to integrate feedback.

Conclusions: The information card can be used by healthcare providers providing counselling to end-of-life care patients and families, including by hospital pharmacists providing discharge counselling.

Project partners have committed to supporting its dissemination.

For the image that goes with this abstract, please see Abstract Appendix, available at https://www.cjhp-online.ca/index.php/cjhp/issue/view/125/ showToc

\section{Pan-Canadian Trends in the Prescribing of Opioids, 2012 to 2016}

Bender M, Cheng R, Sajan P, Gaucher M

Canadian Institute for Health Information, Ottawa, ON

Background: Canada is in the midst of a worsening opioid crisis. Several studies have identified an association between the dispensing of opioids and opioid-related harms. The ongoing monitoring of opioid prescribing trends is needed to support urgent public health surveillance needs to address the crisis.

Objective: To characterize the pan-Canadian and provincial opioid prescribing trends from 2012 to 2016.
Methods: Opioid prescribing trends were characterized by the quantity dispensed as measured by defined daily doses (DDDs), the number of prescriptions dispensed and the number of people who were prescribed opioids. Pan-Canadian aggregate drug claims data were used to examine the number of DDDs and prescriptions dispensed. Additionally, recordlevel drug claims data from three provinces were used to determine the number of people prescribed opioids.

Results: Between 2012 and 2016, the rate of DDDs declined by $9 \%$ from 6,858 to 6,246 DDDs per 1,000 population; while the rate of opioid prescriptions increased by just over $2 \%$, from 582 to 595 prescriptions per 1,000 population. With respect to the number of people prescribed opioids, for the three provinces included in this analysis, the rate decreased from 132 people per 1,000 population to 125 per 1,000 population during the study period. Seniors were prescribed opioids most often with more than 1 in 5 seniors receiving a prescription for an opioid. Among the seniors prescribed an opioid, about 1 in 8 were prescribed a strong opioid on a chronic basis.

Conclusions: The overall quantity of opioids dispensed in Canada declined between 2012 and 2016 despite a steady increase in the number of prescriptions. Moreover, the number of people prescribed an opioid per 1,000 population steadily declined. The results of this study highlight the importance of developing pan-Canadian strategies to reduce the harms associated with the use of prescription opioids.

\section{Cardiac Arrest after Acute on Chronic Colchicine Toxicity}

Keller $D^{1}$, Oesch $A^{1}$, Kelly $L^{1}$, Slessarev $M^{1,2}$

${ }^{1}$ London Health Sciences Centre, London, ON

${ }^{2}$ Western University, London, $O N$

Background: Colchicine is an antimitotic agent with a long history of use in gout and familial Mediterranean fever. For this reason, clinicians may overlook the potentially detrimental effects of colchicine toxicity. Therapeutic, toxic and lethal dose are not well defined and there is no available antidote. We report a case of colchicine use at $0.6-1.2 \mathrm{mg} /$ day in a renally compromised patient which may have contributed to cardiac arrest.

Case Description: An 80-year-old male with renal dysfunction received colchicine $0.6 \mathrm{mg}$ daily as needed and in 2016 , increased to twice daily for 3 days. He decreased back to $0.6 \mathrm{mg}$ daily; however the prescription was not updated. On multiple admissions to hospital over a 1 month period, colchicine was mistakenly increased to twice daily during the medication reconciliation process. On his last admission he presented with diarrhea, nausea, and malaise. His leukocyte count was $0.7 \times 10^{9} / \mathrm{L}$, he was afebrile, and culture negative. Four days later, he was transferred for management of elevated troponin thought to be uremic pericarditis and consideration for intermittent hemodialysis. He was found pulseless and unresponsive while undergoing peritoneal dialysis. Return of spontaneous circulation was achieved after 15 minutes of downtime and he was transferred to intensive care where he later succumbed to his condition.

Assessment of Causality: A Naranjo score of 3 categorized this event as a possible colchicine toxicity owing to increased colchicine dose and subsequent sequelae such as diarrhea, nausea, leukopenia and eventual cardiac arrest. 
Literature Review: Documented risk factors for colchicine toxicity include increased age, renal and/or hepatic dysfunction, chronic use, intravenous use, loading doses, and drug interactions. Clinical stages of toxicity including gastrointestinal, multi-organ failure and recovery phase are also well documented.

Importance to Practitioners: Practitioners should be vigilant with colchicine use and obtaining accurate medication histories due to unpredictable individual dose response and potentially fatal consequences.

\section{Mandatory Quality Related Events Reporting in Canada: A Province-wide Review over 7 Years}

Boucher $A^{1,2}$, Ho $C^{1,2}$, Boyle $T^{3}$, Barker $I^{4}$, MacKinnon $N^{5}$, Zwicker $B^{6}$ ${ }^{1}$ Institute for Safe Medication Practices Canada, Toronto, ON

${ }^{2}$ Leslie Dan Faculty of Pharmacy, University of Toronto, Toronto, $O N$

${ }^{3}$ St. Francis Xavier University, Antigonish, NS

${ }^{4}$ Dalhousie University, Halifax, NS

${ }^{5}$ University of Cincinnati, Cincinnati, $\mathrm{OH}$

${ }^{6}$ Nova Scotia College of Pharmacists, Halifax, NS

Background: There has been limited research that quantitatively analyzes quality related events (QREs) in pharmacies. Recognizing the importance of quality improvement and management to patient safety, several Canadian provinces have moved towards mandatory reporting of QREs in pharmacies to an independent third party.

Description: The objective of this project is to quantify and characterize medication-related QREs that were anonymously reported to a third-party national error-reporting database by pharmacies in a Canadian province over 7 years.

Action: A retrospective analysis was conducted on medication-related QREs from pharmacies occurring between October 1, 2010 and June 30, 2017. Descriptive analysis was performed on all medication-related QREs with respect to type of incident, discoverer, medication system stages, medications, and outcome.

Evaluation: A total of 131, 031 QREs were anonymously reported by 301 pharmacies in Nova Scotia to a third-party national medication safety organization. $74.87 \%(98,097)$ was medication-related QREs. Overall, $82.05 \%(80,488)$ of reported medication-related QREs did not reach the patient (i.e. near misses) and only $0.95 \%(928)$ resulted in harm. Reports of incorrect dose/frequency $(25.58 \% ; 25,089)$, incorrect quantity $(20.00 \% ; 19,619)$, and incorrect drug $(14.22 \% ; 13,951)$ were most common. Pharmacists discovered the majority of medication-related QREs $(75.17 \%$; 73, 739). Order entry was the most frequently reported medication system stage for error occurrence, followed by prescription preparation/dispensing, and prescribing. The most reported medications were levothyroxine sodium, amoxicillin, and rosuvastatin. Medications with the highest proportion of QREs with harm were methadone, risperidone, and warfarin.

Implications: Pharmacists play a significant role in patient safety and preventing medication incidents. The most frequently reported medications were among the top dispensed medications in Canada, but we also identified new high-alert medications. Our findings suggested the need for medication system stage-specific and medication-focused interventions to mitigate harm and improve patient safety.

\section{Analysis of Smart Pump Continuous Quality Improvement Data across Multiple Organizations}

\section{Al-Sukhni M}

Baxter Corporation Canada, Mississanga, $O N$

Background: Smart pump technology can generate Continuous Quality Improvement (CQI) data to provide objective information on IV infusion practices and identify potential safety gaps. However, this valuable data is not frequently shared between organizations.

Objectives: The project's aim was to identify opportunities for increased infusion safety by analyzing smart pump data across organizations nationally.

Methods: A pooled, observational analysis was conducted by pooling smart pump CQI data from five organizations across Canada in November 2016. Data from 7430 pumps was weighted according to the number of pumps per organization and analyzed, by evaluating average drug library compliance, frequency of alerts due to soft or hard limit events and the top 5 drugs with soft and hard limit events. Soft limit alert events consist of overriding the exceeded soft limit or modification of the dose to within soft limits. Hard limit alert events occurred when the set hard limit was exceeded.

Results: An average drug library compliance of 97 percent was found across organizations. No significant variance across patient care areas was noted. Soft limit exceeded alert ranged from 3.6 percent to 11.2 percent of all infusion starts. Hard limit attempted ranged from 1.2 percent to 6.5 percent of all infusion starts. The top five drugs with soft limit events resulting in an override were propofol, hydromorphone, heparin, vancomycin and norepinephrine. The top five drugs with soft limit events resulting in a modification of the dose were morphine, propofol, ondansetron, vancomycin and heparin. The top five drugs with hard limit attempted events were heparin, IV fluids, vancomycin, piperacillin/ tazobactam and amiodarone.

Conclusions: Using smart pump data from multiple organizations nationally provides the opportunity to improve drug safety by focusing on common results, and providing benchmarking information. Changes in drug libraries can also be made to reduce alert fatigue and increase infusion safety.

\section{Comparison of Weight-Based versus Fixed-Dose Norepinephrine Dosing in Patients with Septic Shock}

Azami A, Yrigoyen-DaCruz L, Duronio A

Windsor Regional Hospital, Windsor, ON

Background: Norepinephrine is the first line vasopressor recommended by the 2016 Surviving Sepsis guidelines. There is limited data with respect to superiority of commonly used dosing strategies (fixed versus weight based).

Objective: The primary objective was to compare the two dosing strategies in maintaining target mean arterial pressure (MAP) of $65 \mathrm{~mm}$ $\mathrm{Hg}$ at six hours after initiation of norepinephrine. The secondary objectives were to assess the need for add-on vasopressors, need for hydrocortisone, ICU length of stay, mortality, and treatment side effects.

Method: This was a retrospective, pre-post intervention cohort study. On March 1st 2016, the smart pump programming at this institution changed from fixed-dose $(\mathrm{mcg} / \mathrm{min})$ to weight-based $(\mathrm{mcg} / \mathrm{kg} / \mathrm{min})$. All patients who received norepinephrine six months before and after implementation of this change were screened. Patients who were started 
on norepinephrine and met the 2016 Surviving Sepsis Campaign definition of severe sepsis were included. The primary outcome was analyzed using logistic regression analysis and secondary using descriptive statistics.

Results: Overall 110 patients were included: 54 in the weight-based arm and 56 in the fixed-dose arm. All the baseline characteristics were similar between the two groups. There was a greater proportion of patients at MAP of $65 \mathrm{~mm} \mathrm{Hg}$ or greater at six hours in the weight-based arm compared to the fixed-dose arm $(94.4 \%$ vs. $75.0 \%)$. This corresponded to an odds ratio of 3.5 (95 confidence interval 1.01 to 12.13). There was no statistically significant difference comparing weight-based to fixeddose regimens in: mortality ( $50 \%$ vs. $55 \% ; \mathrm{P}=0.71)$, need for additional vasopressors ( $28 \%$ vs. $45 \%$; $\mathrm{P}=0.18$ ), need for hydrocortisone ( $15 \%$ vs. $20 \% ; \mathrm{P}=0.67$ ), and days spent in ICU (9 days vs. 10 days; $\mathrm{P}=0.26$ ).

Conclusion: Weight-based dosing of norepinephrine is more likely to maintain a MAP of $65 \mathrm{~mm} \mathrm{Hg}$ or greater at six hours compared to a fixed-dose strategy.

\section{Varying Ammonia Levels with Two Formulations of Sodium Phenylbutyrate}

Villanueva J, Lee J

London Health Sciences Centre, London, ON

Background: Ornithine transcarbamylase (OTC) deficiency is a urea cycle disorder treated with ammonia scavenging drugs to prevent hyperammonemic morbidity and mortality. Sodium phenylbutyrate $(\mathrm{NaPB})$ is commercially available as Pheburane ${ }^{\circledR}$ and Buphenyl ${ }^{\circledR}$. This is the first case describing significant variation in ammonia levels with two formulations of $\mathrm{NaPB}$. Pheburane ${ }^{\circledR}$ coated granules can be compounded into a $200 \mathrm{mg} / \mathrm{mL}$ suspension, while Buphenyl ${ }^{\circledR}$ powder is compounded into a $50 \mathrm{mg} / \mathrm{mL}$ suspension.

Case Description: A 3-day-old male was diagnosed with OTC deficiency after ammonia $>400 \mu \mathrm{mol} / \mathrm{L}$ was detected (reference range $<50 \mu \mathrm{mol} / \mathrm{L}$ ). After the initial hyperammonemic crisis was treated, the patient was started on maintenance Pheburane $\AA$, which was compounded into a suspension. The patient's ammonia remained consistently $>100 \mu \mathrm{mol} / \mathrm{L}$ while on Pheburane ${ }^{\circledR}$. Pheburane ${ }^{\circledR}$ was switched to Buphenyl $₫$ after ammonia peaked at $180 \mu \mathrm{mol} / \mathrm{L} 2$ weeks into therapy. The following day, the patient's ammonia drastically declined and remained within normal range for the remainder of therapy.

Assessment of Causality: Although a daily dose of $2.4 \mathrm{~g} \mathrm{NaPB}$ was used with both products, the Pheburane ${ }^{\circledR}$ suspension had a larger volume and was compounded by crushing the coated granules. After reaching peak ammonia levels of $180 \mu \mathrm{mol} / \mathrm{L}$ on Pheburane ${ }^{\circledR}$, the patient was switched to Buphenyl®. Upon the next blood draw fifteen hours later, his ammonia level dropped to $39 \mu \mathrm{mol} / \mathrm{L}$.

Literature Review: A bioequivalence study of subjects aged 19-50 demonstrated that Pheburane ${ }^{\circledR}$ coated granules displayed bioequivalence to Buphenyl®. Moreover, a follow-up study found a reduction in vomiting and hyperammonemic crises when patients switched nitrogenscavenging treatment to Pheburane ${ }^{\circledR}$. Despite this promising evidence, no studies have been published on Pheburane ${ }^{\circledR}$ in neonates.

Importance to Practitioners: Failure to treat neonatal OTC deficiency can lead to coma and death. Clinicians should consider the use of Buphenyl® over Pheburane ${ }^{\circledR}$ when treating neonates and infants.

\section{Stability of $\mathbf{8 . 4 \%}$ Injectable Sodium Bicarbonate When Stored at Room Temperature}

Lam V $V^{2}$, Perks $W^{1}$, Caku $A^{1}$, Walker $S E^{1,2}$

${ }^{1}$ Department of Pharmacy, Sunnybrook Health Sciences Centre, Toronto, ON ${ }^{2}$ Leslie Dan Faculty of Pharmacy, University of Toronto, Toronto, ON

Background: Shortage of sodium bicarbonate injection required the evaluation of alternatives.

Objective: To evaluate the stability of $8.4 \%$ injectable sodium bicarbonate solution in glass vials, compounded in-house and stored at room temperature unprotected from ambient room fluorescent light for 91 days.

Methods: On days 0,2,5,7,9,12,14,16,21,23,28,42,56,70 and 91, a $50 \mathrm{~mL}$ volume $(52.8 \mathrm{~g})$ of sodium bicarbonate solution was withdrawn from each glass vial and titrated against $2 \mathrm{M} \mathrm{HCl}$ to determine buffer capacity. Sodium bicarbonate samples were also delivered to the biochemistry lab for determination of initial $\mathrm{pH}$, sodium, and bicarbonate levels.

Results: Evaluation of titrations of $2 \mathrm{M} \mathrm{HCL}$ against $50 \mathrm{~mL}$ volumes of bicarbonate indicated the method was reproducible. Within day reproducibility for buffer capacity as determined by the standard deviation of regression averaged $0.11 \%$ for $\mathrm{pH} 5$. Similarly, between days variability for sodium and bicarbonate analysis averaged $1.24 \%$ and $1.87 \%$, respectively. The initial $\mathrm{pH}$, before any $2 \mathrm{M} \mathrm{HCl}$ was added, indicates that solutions stored at room temperature increase in $\mathrm{pH}$ by less than $0.05 \mathrm{pH}$ units over the 91 day study period. Analysis of sodium and bicarbonate concentrations indicates less than a 3\% change occurred during the study period and that it would take more than 91 days before a $10 \%$ change was observed, with $95 \%$ confidence. Similarly, analysis of buffer capacity, based on the volume of $2 \mathrm{M} \mathrm{HCl}$ to achieve a $\mathrm{pH}$ of 5 , indicates that it would take more than 10,238 days before a $10 \%$ change was observed and with $95 \%$ confidence this would not occur sooner than 1873 days.

Discussion: We observed that sodium bicarbonate solution, compounded in-house, is stable for at least 91 days, with $95 \%$ confidence, when stored at room temperature. A beyond-use date of 91 days should be used only after due consideration of institutional capability, applicable standards, sterility verification and completion of required quality tests.

\section{Stability of Ropivacaine $2.7 \mathrm{mg} / \mathrm{mL}$, Morphine $0.091 \mathrm{mg} / \mathrm{mL}$ and Ketorolac $0.27 \mathrm{mg} / \mathrm{mL}$ in Polyvinyl Chloride Bags at $4^{\circ} \mathrm{C}$}

Sudbury B, Facca N, Smith $N$

Department of Pharmacy, London Health Sciences Centre, London, ON Leslie Dan Faculty of Pharmacy, University of Toronto, Toronto, ON

Background: The National Association of Pharmacy Regulatory Authorities (NAPRA) Model Standards for Pharmacy Compounding of Non-Hazardous Sterile Preparations indicates that stability data from peer reviewed literature or appropriate experimental studies are required, to assign a beyond use date (BUD) to a given compound.

Objective(s): To determine the stability of ropivacaine $2.7 \mathrm{mg} / \mathrm{mL}$, morphine $0.091 \mathrm{mg} / \mathrm{mL}$ and ketorolac $0.27 \mathrm{mg} / \mathrm{mL}$ in sodium chloride $0.9 \%$ stored in polyvinyl chloride (PVC) plastic bags packaged in an Ultra-Violet Light Inhibiting (UVLI) bag at $4^{\circ} \mathrm{C}$ over 9 days.

Methods: An arthroplasty infiltration solution was prepared and stored in the dark at $4^{\circ} \mathrm{C}$. From day 0 to 9 , samples were taken and frozen at $-85^{\circ} \mathrm{C}$, until analysed by purity-indicating gas chromatography mass spectrometry (GC/MS). In each GC/MS run, chromatographic peaks were generated for the drug and its internal standard. Drug/internal standard peak area ratios were calculated for each analytical run. Peak 
area ratios from quintuplicate test day samples were then calculated as a percentage of the corresponding peak area ratios of quintuplicate day zero samples analysed in the same GC/MS batch. 95\% confidence limits were calculated from these data. Daily physical inspections and $\mathrm{pH}$ measurements were also performed.

Results: The 95\% confidence limits for each measured drug remained above $90 \%$ for the entire study indicating acceptable stability throughout. The arthroplasty infiltration solution remained clear and colourless and the $\mathrm{pH}$ remained essentially constant at a mean of 6.3 with a standard deviation of 0.11 . The mass spectra of the day 9 drug peaks were identical to the corresponding mass spectra of the drug day 0 peaks, demonstrating drug purity to the end of the study.

Conclusion(s): The ropivacaine, morphine and ketorolac arthroplasty mixture in saline prepared in PVC bags stored in the dark at $4^{\circ} \mathrm{C}$ is stable for at least 9 days.

\section{Étude descriptive de la contamination urinaire de travailleurs exposés au cyclophosphamide, à l'ifosfamide, au méthotrexate et au fluorouracile}

Chauchat $L^{1}$, Therrien $R^{2}$, Dufour $A^{3}$, Gagné $S^{4}$, Caron $N^{4}$, Bussières $J F^{1,5}$ ${ }^{1}$ Unité de recherche en pratique pharmaceutique, Département de pharmacie, Centre Hospitalier Universitaire Sainte-Justine, Montréal, QC ${ }^{2}$ Département de pharmacie, CISSS de Laval, Laval, QC

${ }^{3}$ Département de pharmacie, CISSS de la Montérégie-Centre, Greenfield Park, QC

${ }^{4}$ Centre de toxicologie du Québec, Institut national de santé publique du Québec, Québec, QC

${ }^{5}$ Faculté de pharmacie, Université de Montréal, Montréal, QC

Contexte : Certains professionnels de la santé sont exposés aux médicaments dangereux et une absorption systémique de ces médicaments est possible.

Objectif : Déterminer la faisabilité d'un programme de surveillance biologique par détection de médicaments dangereux.

Méthodologie : Étude descriptive observationnelle. Cohorte d'infirmiers, pharmaciens et assistants-technique manipulant des médicaments dangereux au sein de deux centres d'oncologie adultes (A (750 lits) et B (700 lits)). Ont été mesurés : cyclophosphamide (limite de détection: $0,009 \mathrm{ng} / \mathrm{mL})$, ifosfamide $(0,0097 \mathrm{ng} / \mathrm{mL})$, méthotrexate $(0,075 \mathrm{ng} / \mathrm{mL})$ et FBAL, métabolite urinaire du fluorouracile $(0,120 \mathrm{ng} / \mathrm{mL})$ par UPLC/MS-MS. Chaque participant volontaire a fourni un échantillon d'urine en fin de journée et complété un journal.

Résultats : Ont été recrutés 56 participants ayant au moins une activité liée à la manipulation de médicaments dangereux ciblés soit 27/28 (A) et 24/28 (B). En centre A, l'utilisation des gants était conforme pour $77 / 89(86,5 \%)$ des activités réalisées par 13 infirmiers. Deux infirmiers $(2 / 13)$ portaient une blouse et aucun (0/13) le masque pendant l'administration. Tout le personnel de la pharmacie (15/15) portait les protections recommandées pour l'ensemble des activités (34/34). En centre B, l'utilisation des gants était conforme pour 49/68(72\%) des activités réalisées par 10 infirmiers. Neuf infirmiers (9/10) portaient une blouse et quatre $(4 / 10)$ portaient un masque pendant l'administration. Les assistants techniques portaient l'équipement recommandé pour la préparation et manipulation des médicaments dangereux en salle blanche (3/3). Seulement deux pharmaciens (2/8) et deux assistants techniques (2/9) portaient l'ensemble de l'équipement recommandé pendant leurs activités. Aucun échantillon (0/56) ne comportait de trace détectable des quatre médicaments dangereux.
Conclusion : Il est faisable de mettre en place un programme de surveillance biologique en établissement de santé. Un tel programme doit comprendre un profil des mesures de protection appliquées par travailleur exposé. Cette étude confirme l'absence de traces de quatre médicaments dangereux dans l'urine de 56 travailleurs en oncologie adulte.

\section{Stability of 10, 40 and $200 \mathrm{mcg} / \mathrm{mL}$ Hydromorphone Solutions Stored in Syringes at Room Temperature $\left(23^{\circ} \mathrm{C}\right)$}

Hook $R^{1}$, Riss $V^{1}$, Scharrer $E^{1}$, Neault $A^{1}$, Law $S^{2}$, Walker $S E^{2,3}$

${ }^{1}$ Departments of Pharmacy, Hospital for Sick Children, Toronto, ON

${ }^{2}$ Sunnybrook Health Sciences Centre, Toronto, ON

${ }^{3}$ Leslie Dan Faculty of Pharmacy, University of Toronto, Toronto, ON

Background: Inpatient hospital pharmacies must compound intravenous products and assign an appropriate beyond-use-date (BUD) as per NAPRA standards, because products are not commercially available. Having opioid infusions available as ready-to-administer (RTA) products on nursing units is important for safe and timely administration of pain control. Paediatric patients require lower concentrations of opioid continuous infusions than adults and previous publications have demonstrated the stability of hydromorphone, but not at lower concentrations and not in syringes.

Objective: We sought to evaluate the chemical stability of lower concentrations of hydromorphone, prepared in syringes

Methods: On study day $0,50 \mathrm{~mL}$ solutions of $0.2 \mathrm{mg} / \mathrm{mL}$ and $10 \mathrm{mg} / \mathrm{mL}$ concentrations of hydromorphone were prepared in $\mathrm{BD}$ syringes. 3 units of each container and concentration were stored at room temperature. Concentration analysis was completed on study days $0,1,3,7,14,24$, $38,51,78$ and 90 . Hydromorphone concentrations were determined by a validated stability-indicating liquid chromatographic method with UV detection. Chemical stability was based on the intersection of the lower limit of the $95 \%$ confidence interval of the observed degradation rate and the time to achieve $90 \%$ of the initial concentration.

Results: The analytical method separated degradation products from hydromorphone such that the concentration was measured specifically, accurately (deviations from known averaged $2.1 \%$ ) and reproducibly (replicate error was less than $0.6 \%(\mathrm{CV}(\%))$. During the study period all solutions retained more than $98 \%$ of the initial concentration. Analysis of variance revealed significant differences in percent remaining due to concentration $(\mathrm{p}<0.001)$, but not study day $(\mathrm{p}=0.339)$. The study was capable of detecting a $0.81 \%$ difference in concentration due to study day or concentration. The calculated beyond-use-date exceeded the 90-day study period for all concentrations.

Conclusions: We conclude that 10,40 and $200 \mathrm{mcg} / \mathrm{mL}$ solutions of hydromorphone diluted in saline and stored in $\mathrm{BD}$ polypropylene syringes are physically and chemically stable for at least 90 days at room temperature $\left(23^{\circ} \mathrm{C}\right)$

\section{Atrial Fibrillation Patient Knowledge Gaps: A Systematic Review}

Salmasi S, Loewen P

Faculty of Pharmaceutical Sciences, University of British Columbia, Vancouver, $B C$

Background: Atrial fibrillation (AF) patients frequently do not adhere to their medication. Poor patient understanding of their disease has been associated with poor adherence. Identifying patients' knowledge gaps is the first step towards improving patient education strategies, adherence 
and health outcomes. To date, there are no systematic reviews of AF patient knowledge gaps.

Objective: To characterize the published literature about AF patients' knowledge of their condition and medications, and to identify knowledge gaps.

Methods: Systematic review following PRISMA guidelines. We systematically searched PubMed, Embase, CINAHL and PSYCHINFO for quantitative and qualitative studies that measured people's knowledge about $\mathrm{AF}$, stroke, rate/rhythm control and stroke prevention medications using "AF patient knowledge" and closely-related terms. Qualitative data was summarized narratively. We grouped quantitative data from related questions into "knowledge categories", calculating the median and IQR of the $\%$ correct responses for each category. A category with median $\leq 50 \%$ was classified as a "knowledge gap". Quality was assessed using standard design-specific quality of reporting appraisal instruments.

Results: We included 19 studies involving 4,582 participants, with 89\% being of high quality. Less than half of the studied patients were aware that $\mathrm{AF}$ can be asymptomatic, can recur, or predispose to heart failure and stroke. Other knowledge gaps included: AF causes, symptoms, the rationale for oral anti-thrombotic (OAT) therapy, and OAT- related dietary restrictions. AF patients' knowledge of stroke signs/symptoms, and rate/rhythm medications could not be assessed due to paucity of data.

Conclusions: Majority of patients lack knowledge of AF causes, symptoms, risk of stroke, the rationale for anti-thrombotic therapy and drug and food interactions. Our results highlight the need for more widespread and better-quality patient education strategies.

\section{A Feasibility Study: Implementing a Medication Adherence Contract in Kidney Transplant Recipients Followed by an Outpatient Transplant Clinic}

Desai $A^{1}$, Burger $C^{1}$, Wallace $C^{1}$, Treleaven $D^{2}$, Shipley $A^{1}$

${ }^{1}$ Pharmacy Department, St. Joseph's Healthcare Hamilton, Hamilton, ON

${ }^{2}$ Division of Nephrology, Department of Medicine, St. Joseph's Healthcare

Hamilton, $O N$

Background: Medication adherence contracts have been more effective in improving adherence compared to providing educational pamphlets, pillboxes and medication refill reminders alone.

Objectives: Primary objective was to determine patient enrollment. Secondary objectives investigated patient retention, time required for encounters, interventions by pharmacists, patient satisfaction, health care professional acceptability and perception of patient adherence to immunosuppressants.

Methods: Kidney transplant recipients transplanted within 0-6 months, followed by the Outpatient Transplant Clinic, with access to a telephone that could be communicated to in English were approached for consent. The Contract was administered in clinic or Inpatient Transplant unit using semi-structured discussions and reviewed in 3 to 9 weeks with patients via phone.

Results: Seventeen of 47 eligible patients (36\%) were enrolled over 3.3 weeks in the clinic $(n=11)$ and on the inpatient unit $(n=6)$. Patient enrollment was $52 \%$ (17 of 33 patients) considering only 33 of 47 eligible patients were approached. Barriers to enrollment were back to back clinic appointments, lack of designated clinic space and prioritizing non-research related responsibilities during the last 1-2 weeks of data collection. Median time for administration and review of the Contract was 21 and 12 minutes respectively. Interventions provided include discussion of adherence tools $(n=13)$; education on immunosuppressants $(n=6)$, consequences of nonadherence $(\mathrm{n}=5)$ and anti-infective medications $(\mathrm{n}=4)$ as well as medication regimen simplification $(n=5)$. Most patients found their encounters with pharmacists valuable $(n=6)$ or extremely valuable $(n=6)$. More than half of health care professionals were very likely to identify and refer patients that may benefit from a Medication Adherence Contract $(n=8)$.

Conclusion: Medication Adherence Contracts are feasible in practice provided there is dedicated space and time for encounters. Majority of patients and professionals were satisfied with and accepting of the Contract respectively. This study provides recommendations for those considering adopting such contracts in practice.

\section{An Emerging Challenge: Pharmacists' Ability to Counsel a Growing Immigrant Population}

Jalal $A$

Leslie Dan Faculty of Pharmacy, University of Toronto, Toronto, ON

Background: Counselling provided by pharmacists is a critical component for the safe and effective use of medications, which is particularly important given the serious consequences of errors. In the case of new international migrants, however, cultural barriers might be limiting pharmacists' ability to counsel effectively.

Objectives: The study aimed to identify: cultural factors noted in the literature to influence interactions between pharmacists and immigrants during counselling sessions; and potential solutions to cultural barriers in pharmacy counselling.

Methods: A scoping review was conducted, which consists of the following 5 stages: (1) identifying the research questions, (2) identifying the relevant studies, (3) identifying criteria for study selection, (4) charting the data, and (5) collating the results. The quality of selected studies was also assessed.

Results: Only 7 studies were found, but revealed 4 important factors: preconceived beliefs about drugs and healthcare, language barriers, use of informal interpreters, and nonverbal communication. Several solutions for reducing medication errors and increasing patient safety were also identified. One solution is to work towards achieving a certain level of understanding about ethnic minority patients. This involves increasing cultural sensitivity among pharmacists and learning about immigrant patients' culture and illness perception.

Conclusion: The literature is sparse, but confirms that assessment and redress of cultural disparities is important to improve medication use and the quality of encounters with patients of all cultural backgrounds.

\section{Toxicology Education Needs of Emergency Department Pharmacists}

Dewaal C, Mink M

Alberta Health Services, Calgary, $A B$

Background: Emergency department (ED) pharmacists are routinely consulted on the care of poisoned patients. Toxicology training varies by country and the learning needs of pharmacists have not been explored.

Objective: To survey and compare subjective toxicology knowledge and learning needs of ED pharmacists in Canada and the United States (US).

Methods: Pharmacists were surveyed via online emergency medicine speciality networks hosted by the Canadian Society of Hospital Pharmacy and American College of Clinical Pharmacy. Pharmacists rated their knowledge and interest for future learning regarding 29 toxicology topics using a 5-point Likert scale. Preferred learning formats were also surveyed. Paired T-tests were conducted to assess knowledge and interest differences between US and Canadian pharmacists. 
Results: Two-hundred ED pharmacists (response rate 16\%) responded to the survey. Pharmacists rated their knowledge highest on analgesics and communicating with poison centres. Chloroquine, metals and bioterrorism were rated as the least knowledgeable subjects. Pharmacists identified drugs of abuse, blood gas and ECG interpretation as the most desired topics for future learning. Respondents were least interested in learning to communicate with poison centres, and about chloroquine and theophylline poisonings. Webinars and podcasts were the preferred learning formats for pharmacists. Online discussion forums, YouTube videos and toxicology rotations were the least popular. US pharmacists consistently rated their knowledge higher than Canadian pharmacists (Mean Difference in Likert Scores 0.58, 95\% CI: 0.48-0.69). No significant difference was noted in interest levels between the two groups. Conclusions: Future education for ED pharmacists should focus on diagnostic assessment of the poisoned patient and drugs of abuse. Delivery should be in webinar format. Toxicology residencies and fellowships are available in the US and may reflect differences in knowledge assessment scores.

\section{Implementation of an Overnight Pharmacist Model}

Charron $M^{1}$, Chen $E^{1}$, Bombassaro AM ${ }^{1,2}$, Harris $V^{1,2}$, Newman $J^{1}$

${ }^{1}$ London Health Sciences Centre, London, ON

${ }^{2}$ Western University, London, $O N$

Background: The Pharmacy Department at a tertiary care academic centre initiated overnight pharmacist services in 2014 in response to computerized physician order entry.

Description: One of the hospital sites developed an overnight model in which 2 pharmacists rotate every 2 weeks through weeknight shifts (2230 to 0630 hours) and weekday clinical services to a surgical unit. The pharmacists cross cover each other for vacations. Comprehensive overnight services include order verification, therapeutic interventions and drug information. Daytime pharmacists are scheduled to cover weekend overnight services.

Action: After surveying 24 hour operations at select Ontario hospitals a site-specific model was created through a consensus process. The goal was to create a role that optimized professional satisfaction, recruitment and retention. This was accomplished by ensuring opportunities similar to those of daytime pharmacists with respect to direct patient care, education, teaching and research with comparable scheduling percentages for distribution and clinical time. Two pharmacists were recruited and intensively trained over 3 months.

Evaluation: During a weeknight shift the pharmacist on average verifies 500 medication orders for 650 patients, of which 25 are new admissions, thus alleviating the morning workload and alerting daytime pharmacists to patients needing prioritized pharmaceutical care. The pharmacists are clinically integrated into a surgical service, which previously had high pharmacist turnover, with excellent feedback regarding the consistent quality of service. Weekly discussions take place with the clinical practice leader regarding patient cases and role feedback. Job satisfaction and retention have been positive with approximate 3 year tenure for the current team. A third pharmacist has been successfully recruited into the role.

Implications: This successful service model, as evidenced from operational and clinical perspectives, can be used as a template for other hospitals seeking to implement or modify an overnight pharmacist role, particularly if retention has been challenging.

\section{A Literature-Based Algorithm for the Assessment, Management and Monitoring of Drug-Induced QTc Prolongation in the Psychiatric Population}

Zolezzi $M^{1}$, Cheung $L^{2}$

${ }^{1}$ College of Pharmacy, Qatar University, Doha, Qatar

${ }^{2}$ Grey Nuns Hospital, Edmonton, $A B$

Background: Certain psychotropics and a number of other medications used to treat medical conditions in psychiatric patients can increase the risk of prolonging the corrected QT (QTc) interval on the electrocardiogram which poses patients at risk of life-threatening ventricular arrhythmias such as Torsades de Pointes. Pharmacists are often consulted about medications which are known to prolong the QTc interval. Although this information is often accessible, advising how to identify, assess, manage and refer psychiatric patients at risk for drug-induced QTc prolongation is more challenging.

Objectives: The objective of this project was first to review the literature which describe guidelines and recommendations for the assessment and management of drug-induced QTc prolongation, and then to design an algorithm to be used by pharmacists working closely with mental health professionals or who provide care to psychiatric patients.

Methods: A review of the literature was undertaken. Predefined keywords were used to perform the database search in MEDLINE, EMBASE, and International Pharmaceutical Abstracts to identify reviews, reports and guidelines on the assessment, prevention or monitoring of drug-induced QTc prolongation emphasizing on psychotropic medications and management in the psychiatric population.

Results: A total of 560 relevant citations were retrieved from the electronic database search, of which a total of 22 relevant articles were selected. Of these, only 8 articles provided recommendations for the assessment and management of drug-induced QTc prolongation in psychiatric patients. Although the remainder 14 articles did not discuss strategies specifically for the psychiatric population, they were still reviewed and some relevant recommendations adapted in the development of the algorithm and related guide.

Conclusion: The literature-based algorithm developed provides a stepped based approach for the assessment, management and monitoring of drug-induced QTc prolongation in the psychiatric population. After this developmental phase, feedback on the algorithm and related guide by pharmacists will be undertaken.

\section{Provincial Smart Pump Program Improves Patient Safety}

Bunker $J^{1}$, Herod $C^{1}$, Lyons $B^{2}$, Sellinger $D^{1}$

${ }^{1}$ Regina Qu'Appelle Health Region, Regina, SK

${ }^{2}$ Prairie North Health Region, North Battleford, SK

Background: The use of Dose Error Reduction Software to inform pump programmers of potential keystroke, entry errors or potentially harmful doses reduces patient harm. The creation of a single province-wide drug library ensures the same level of safety across the province and facilitates patient movement between sites.

Description: A multi-disciplinary team from all Regional Health Authorities and the Cancer Agency developed the drug library and implemented SMART pumps throughout the entire province. A provincial program team was created to: maintain and update the drug library, maintain provincial parenteral monographs, and report on regional and provincial use of smart pumps. 
Action: Once the smart pump was implemented in all health regions, the Provincial Smart Pump Program was established to maintain, update and report on the use of smart pumps. A monthly drug library update cycle was established during the first year. Updates from the Program are delivered to regional servers where local staff update their smart pumps with the newest drug library.

Evaluation: Six percent of 2,351,379 program attempts created an alert; $2 \%$ of programs were edited based on the alert resulting in 46,213 program changes. Each program change may have avoided harm if infused as originally programmed. The 10 Medication lines were responsible for 34,699 alerts in 315,158 program attempts. Of these, 11,296 alerts resulted in a programming change.

Implications: Province-wide standardization of the drug library, medication preparation and parenteral monographs facilitates the safe transfer of patient throughout the continuum of care across the province without wastefully re-preparing infusions for local protocols. Work standards ensure regions continue to utilize the most current drug library. The Smart Pump Program evaluates the drug library and alerts, with feedback from users, for appropriateness. Reporting can highlight regional practice variation for investigation. Dose limit notifications reduce potential patient harm from infusions.

\section{A Multi-Incident Analysis on Medication Incidents Associated with Patient Harm}

Boucher $A^{1,2}$, Dhanjal $S^{1,3}$, Ho $C^{1,2,3}$

${ }^{1}$ Institute for Safe Medication Practices Canada, Toronto, ON

${ }^{2}$ Leslie Dan Faculty of Pharmacy, University of Toronto, Toronto, ON

${ }^{3}$ School of Pharmacy, University of Waterloo, Waterloo, $\mathrm{ON}$

Background: Medication incidents associated with patient harm can either result in sub-optimal disease management or expose patients to unnecessary life-threatening situations, calling for attention to such incidents and the need to adopt strategies to improve overall patient and medication safety.

Description: The objective of this multi-incident analysis was to gain a deeper understanding of the possible contributing factors to incidents associated with patient harm, and to develop potential recommendations to prevent error recurrences.

Action: A total of 971 medication incidents associated with patient harm were extracted from a national incident reporting database from 2009 to 2017, with the subsequent performance of a qualitative and thematic analysis on 909 incidents that met the inclusion criteria.

Evaluation: Three main themes were identified from the multi-incident analysis, which included (1) high-risk processes in pharmacy practice, (2) communication gaps, and (3) preventable adverse drug reactions. Subthemes were further developed for each theme, which included (1) methadone maintenance therapy, (2) multi-medication compliance aids, and (3) compounding; (1) patient-provider engagement and (2) interprofessional collaboration; and lastly, (1) drug-drug interactions and (2) documented drug allergies.

Implications: Independent double checks can be considered as a gate-keeping strategy for high-risk processes that are involved in the medication-use system. Clear communication within the circle of care is crucial for safe and effective patient-centered care. Technology can serve as clinical decision support for healthcare practitioners in mitigating preventable adverse drug reactions. It is hoped that findings from this analysis and potential solutions presented can aid with the adoption of error reduction strategies and safe medication practices. Sharing lessons learned from medication incidents will contribute to overall patient and medication safety.

\section{Insourcing High-Risk Sterile Compounded Injectables: Development of In-House Capacity to Produce High-Quality Injectables during the Sodium Bicarbonate Shortage}

Perks W, Caku A, Lye M, DeFigueiredo S, Wun B, Walker SE

Department of Pharmacy, Sunnybrook Health Sciences Centre, Toronto, ON University of Toronto, Toronto, $\mathrm{ON}$

Background: High-risk sterile compounding appears to have decreased considerably in recent years given perceived risk and regulatory/media attention. When commercial supplies are lacking and where alternatives are inadequate, Pharmacies need to weigh patient risk from lack of access against their ability to prepare a high quality compounded sterile product (CSP).

Description: Formulation of a CSP - Sodium Bicarbonate 8.4\% Injection (SBI) was undertaken during disruption of commercial supply and used to develop the infrastructure to extend this capability to other future CSPs.

Action: Specifications utilizing USP were developed for our SBI formulation. Multiple test iterations were prepared until the desired elements were attained. A "Quality by Design" (QBD) approach was utilized in the formulation process which attempts to mitigate against foreseeable risk elements during production. Validation and testing of the finished product for endotoxin level and sterility were completed through an outside laboratory and in-house quality testing for particulates, $\mathrm{pH}$, sodium and bicarbonate concentrations were completed for each batch.

Evaluation: Development of the SBI and the QBD approach required approximately 0.25 FTE each of Pharmacist and Pharmacy Technician time. Time to implementation of a patient-ready CSP was approximately 3 months from inception. Batch production quantity models for best economic return were developed based on expected monthly use and batch costs. The final SBI prepared lots met all USP specifications for Sodium/Bicarbonate content, $\mathrm{pH}$, endotoxin levels and sterility.

Implications: By following a QBD approach, Pharmacies can prepare a high-quality injectable from raw non-sterile starting ingredients. Appropriate final product quality testing should be undertaken. Up-front investments and effort are required in order to safely develop the required resources for safe compounding, but enhances patient access to necessary products in the event of commercial supply disruption or inadequate therapeutic alternatives.

\section{Coordinating a Response to a Critical Drug Shortage: Experience with Sodium Bicarbonate}

Mysak T, Slobodan J, Simpson T, Horon K, Lazarenko G Pharmacy Services, Alberta Health Services, $A B$

Background: In June 2017, a critical shortage of intravenous sodium bicarbonate occurred across Canada. Our health authority needed to act rapidly to ensure availability of a drug used in critically ill patients.

Description: Sodium bicarbonate as an intravenous solution is provided by a single supplier with important therapeutic value in critically ill patients. When production issues, compounded by a voluntary recall, rapidly depleted the stock on hand and hampered any ability to procure more, our heath authority faced a situation of having less than one week's stock on hand. At the time, usage patterns were approximately 2000 vials per week. No commercially produced or compounded product was available and Health Canada would not rapidly commit to allowing importation of foreign supply. 
Action: Our senior pharmacy leadership team ran a virtual Emergency Operations Centre (EOC) to coordinate efforts across our sites and report up to senior organizational leadership. EOC members met with clinical stakeholders to determine restrictions on sodium bicarbonate use, coordinated communication to all sites and impacted departments, validated and tested a recipe for compounding, worked out a process for sterile filtration of potentially contaminated product, and worked with senior organizational leadership to prioritize the various strategies developed to manage the crisis.

Evaluation: Weekly stock counts and utilization reporting at all sites demonstrated that restricting product use resulted in an approximate $75 \%$ reduction in overall consumption. This rate was maintained for the duration of the shortage and may point to potential ongoing cost savings. Follow-up with clinical stakeholders suggested minimal negative patient impact from the restrictions.

Implications: By leveraging our central governance and coordination efforts, our pharmacy team was able to overt a potentially critical situation. Lessons learned for future shortages include exploration of our internal compounding resources and ability to identify inappropriate use of drugs in our health authority.

\section{Development and Implementation of a Mobile Antimicrobial Handbook App at a Tertiary Teaching Hospital}

Lo $J^{1}$, Wan $M^{1}$, Lepore $J^{2}$, Elligsen $M^{1}$, Walker $S A N^{1,3,4,5}$, Leis $J A^{4,5,6}$ ${ }^{1}$ Department of Pharmacy, Sunnybrook Health Sciences Centre, Toronto, ON ${ }^{2}$ Digital and Visual Communications Department, Sunnybrook Health Sciences Centre, Toronto, ON

${ }^{3}$ Leslie Dan Faculty of Pharmacy, University of Toronto, Toronto, ON

${ }^{4}$ Division of Infectious Diseases, Sunnybrook Health Sciences Centre, Toronto, ON

${ }^{5}$ Sunnybrook Health Sciences Centre Research Institute, Sunnybrook Health Sciences Centre, Toronto, ON

${ }^{6}$ Faculty of Medicine, University of Toronto, Toronto, $O N$

Background: Institutional antimicrobial guidelines were previously available as printed and electronic format handbooks. With the ever increasing use of technology to improve direct patient care, our hospital's Antimicrobial Stewardship Team (AST) recognized the need to optimize the accessibility and usability of our current guidelines.

Description: In June 2015, our AST began developing an Antimicrobial Handbook app for mobile devices and desktop computers. This electronic tool includes treatment and prophylaxis guidelines, annual hospital antibiograms, renal dosing guidelines, and safety guidelines for pregnancy and breastfeeding.

Action: The mobile site was launched in November 2015, and promoted to pharmacists and physicians at our institution and other practice sites. The app content and format are continuously reviewed and updated based on advances in clinical evidence and feedback from its users. Quantitative user data is electronically provided monthly from Google Analytics Solutions.

Evaluation: Between January 1, 2017 and June 30, 2017, there have been over 10,000 page views of our app, by over 2,000 unique users. There has been a $73 \%$ increase in usage, with an average of 1,720 visits per month to the homepage in 2017, compared to 995 visits per month in 2016. Similarly, the number of total users and new users has increased by $100 \%$ and $84 \%$, respectively. The majority of our users are located in the Greater Toronto Area (75\%), but our app also has frequent users from Hamilton,
Ottawa, Montreal, and other Canadian cities. The most visited sections are the treatment guidelines (37\%), antimicrobials (27\%), and renal dosing guidelines (14\%).

Implications: The Antimicrobial Handbook app is an extremely innovative tool that has provided widespread access to clinically relevant information, enabled efficient information updates, and allowed for timely, accurate evaluation to assess the need for ongoing modifications. Evaluation of the clinical impact of this app on antimicrobial prescribing practices is planned.

\section{Retrospective Multicentre Cohort Study Comparing Safety and Efficacy Outcomes with Intermittent Infusion versus Continuous Infusion Vancomycin \\ Ma $N^{1,2}$, Walker $S A N^{1,2,3,4, *}$, Elligsen $M^{1}$, Palmay $L^{1}, H o G^{5}$, Leis JA, Bansal $V^{6}$, Powis 5 \\ ${ }^{1}$ Department of Pharmacy, Sunnybrook Health Sciences Centre, Toronto, ON ${ }^{2}$ Leslie L. Dan Faculty of Pharmacy, University of Toronto, Toronto, ON \\ ${ }^{3}$ Division of Infectious Diseases, Sunnybrook Health Sciences Centre, \\ Toronto, ON \\ ${ }^{4}$ Sunnybrook Health Sciences Centre Research Institute, Sunnybrook Health Sciences Centre, Toronto, ON \\ ${ }^{5}$ Michael Garron Hospital, Toronto, ON \\ ${ }^{6}$ Holland Centre, Sunnybrook Health Sciences Centre, Toronto, ON \\ * Senior Author; sequence determines credit approach to authorship}

Background: Patients with good renal function receiving intermittent infusion vancomycin (IIV) may require total daily doses $\geq 4 \mathrm{~g}$ to achieve trough concentrations of $15-20 \mathrm{mg} / \mathrm{L}$, increasing the risk of vancomycin associated nephrotoxicity (VAN). Continuous infusion vancomycin (CIV) may enable attainment of concentrations between $15-20 \mathrm{mg} / \mathrm{L}$ with a lower daily vancomycin dose, potentially reducing the risk of VAN.

Objectives: The primary objective was to compare VAN risk (serum creatinine $(\mathrm{sCr})$ increase $\geq 50 \%$ from baseline) and renal damage $(\mathrm{sCr}$ increase $\geq 100 \%$ from baseline) in patients receiving IIV versus CIV. The secondary objective was to compare clinical cure between IIV and CIV in patients who achieved vancomycin trough or steady state concentrations of $\geq 15 \mathrm{mg} / \mathrm{L}$, respectively.

Methods: Retrospective chart reviews for eligible patients admitted to Sunnybrook Health Sciences Centre or Holland Orthopaedic and Arthritic Centre between January 1, 2010 and December 31, 2016 were completed. Adult inpatients who received at least 48 hours vancomycin for a documented gram positive infection and had at least one steady state vancomycin concentration were eligible. Baseline patient characteristics, safety and efficacy outcomes for the IIV and CIV cohorts were compared using appropriate statistical tests (Fisher's exact, Student's t-test, or MannWhitney), with significance defined as $\mathrm{P}<0.05$.

Results: Of 2134 patients identified, 1104 (52\%) met inclusion criteria. Chart review has been completed for 89 patients (113 courses of vancomycin). Patients receiving courses of IIV were more likely to be at risk of VAN (15/62 [24.2\%] versus 4/51[7.8\%]; $\mathrm{P}=0.02)$ and experience renal damage (9/62 [14.5\%] versus $1 / 51$ [2.0\%]; $\mathrm{P}=0.02)$. There was no difference in clinical cure between IIV (19/27 [70.4\%]) and CIV patients (13/17 [76.5\%]; P=0.74).

Conclusion: Patients in the IIV cohort were more likely to experience increases in $\mathrm{sCr}$ resulting in VAN risk and renal damage. The results indicate there is no difference in clinical cure between patients who received IIV versus CIV. 


\section{Evaluation of Dosing Strategies with Meropenem using Monte Carlo Simulations against Bacteria with Raised MIC}

Moscoso $D^{1,2}$, Walker $S A N^{1,2,3,4 *}$

${ }^{1}$ Department of Pharmacy, Sunnybrook Health Sciences Centre, Toronto, ON

${ }^{2}$ Leslie L. Dan Faculty of Pharmacy, University of Toronto, Toronto, ON

${ }^{3}$ Division of Infectious Diseases, Sunnybrook Health Sciences Centre,

Toronto, $O N$

${ }^{4}$ Sunnybrook Health Sciences Centre Research Institute, Sunnybrook Health Sciences Centre, Toronto, $O N$

*Senior Author; sequence determines credit approach to authorship

Background: The worldwide increased prevalence of gram negative bacterial (GNB) resistance coupled with few therapeutically appropriate available antibiotics (TAAAs) necessitates evaluation of dosing strategies to optimize the use of TAAAs against more resistant pathogens. Burn patients are a unique population, having altered pharmacokinetics for many antibiotics and high risk of infection with resistant GNB, to add increased complexity to their care.

Objective: The objective of this study was to identify optimal dosing of meropenem in burn patients using pharmacokinetic and pharmacodynamic (PK-PD) targets for meropenem against bacteria with an elevated minimum inhibitory concentration (MIC).

Methods: Weighted mean pharmacokinetic parameters for meropenem in burn patients and healthy volunteers were determined from the published literature. Monte Carlo simulations (MCS) with 1,000,000 iterations were completed to determine the probability of target attainment (PTA) for several intermittent infusion (IIV) and continuous infusion (CIV) meropenem dosing regimens using weighted mean pharmacokinetic parameters and MICs ranging from 0.5 to $32 \mathrm{mg} / \mathrm{L}$ for burn patients and healthy subjects (HS). PK-PD targets evaluated were $\%$ time above MIC (\%T>MIC) of 30\%, 50\% and 80\%.

Results: Compared to HS, burn patients had an increased meropenem volume of distribution (V), and decreased elimination rate constant $(\mathrm{k})$, resulting in a calculated clearance $(\mathrm{Cl})$ that was comparable to $\mathrm{HS}$ $(\mathrm{Cl}=\mathrm{kV})$. PTA in burn patients was different than in $\mathrm{HS}$ with different meropenem dosing regimens due to differences in $\mathrm{V}$ and $\mathrm{k}$ between these patient populations. At higher MICs, CIV regimens had a better PTA than IIV in HS and burn patients. Both meropenem CIV $1 \mathrm{~g} \mathrm{IV} \mathrm{q4h} \mathrm{and}$ $2 \mathrm{~g}$ IV q8h provided optimal PTA with a high MIC of up to $8 \mathrm{mg} / \mathrm{L}$.

Conclusion: In burn patients with resistant GNB up to an MIC of $8 \mathrm{mg} / \mathrm{L}$, the optimal PTA is achieved with continuous infusion meropenem of $1 \mathrm{~g}$ IV q4h or $2 \mathrm{~g}$ IV q8h.

\section{Development of an HIV National Clinical Observership Program for Pharmacists}

Tseng $A^{1,2}$, Yoong $D^{3}$, Foisy $M M^{4}$, Giguere $P^{5}$, Robinson $L^{6}$, Stuber $M$, on behalf of the Canadian HIV and Viral Hepatitis Pharmacists Network (CHAP)

${ }^{1}$ University Health Network, Toronto, ON

${ }^{2}$ Leslie Dan Faculty of Pharmacy, Toronto, $O N$

${ }^{3}$ St. Michael's Hospital, Toronto, $O N$

${ }^{4}$ Northern Alberta Program, Royal Alexandra Hospital, Edmonton, AB

${ }^{5}$ The Ottawa Hospital, Ottawa, ON

${ }^{6}$ Windsor Regional Hospital, Windsor, $O N$

${ }^{7}$ Regina Qu'Appelle Health Region, Regina, SK

Background: Pharmacists play a significant role in optimizing care for HIV patients, managing challenges of adherence, resistance, comorbidities, polypharmacy and drug interactions. HIV residency programs or electives provide specialized training but may not be ideal for practicing pharmacists. A national observership program was developed to meet the learning needs of working pharmacists.

Description: The observership program was launched in 2017 by a national network of HIV pharmacists. Program objectives were to improve pharmacists' confidence in HIV therapy management, to increase awareness of different practice sites and subspecialties, and to promote collaboration. All pharmacists wishing to gain clinical experience or specialized knowledge through shadowing/teaching with another HIV pharmacist were eligible to apply. Observerships of 1 to 5 days in duration were offered.

Action: A working group developed terms of reference and application criteria. Funding was secured for one year via an unrestricted industry educational grant. Calls for applications were issued through the network, with priority given to new practitioners or those from rural/ underserviced areas.

Evaluation: To date, 4 observerships have been completed with all participants completing surveys on their experience. On average, observers had worked for 4 years, with less than 6 months of experience providing HIV care in a hospital (50\%) or clinic/community (50\%) setting; 75\% were practicing in a small urban centre. Overall, pharmacists found the observership to be extremely beneficial, and resulted in increased confidence in therapeutic knowledge and ability to provide care and enhanced professional networking with potential for future collaboration. All preceptors felt the workload was reasonable and were willing to offer future observerships.

Implications: A national clinical observership program has been successful in providing learning and mentorship opportunities for HIV pharmacists. Continued program funding is being pursued. This program may serve as a model for implementation in other countries or in other therapeutic areas.

\section{An Innovative In-House Developed Access ${ }^{\circledR}$ Database to Capture and Analyze Antimicrobial Stewardship Interventions}

\section{Patel S, Patel $M$}

Humber River Hospital, Toronto, ON

Background: Antimicrobial stewardship programs are responsible for optimizing use of antimicrobials. Our program focuses on reviewing antimicrobials on Day 3. Infectious disease physician and pharmacist are responsible to review antimicrobials on clinical care areas. We utilize a Meditech system, which provides antimicrobial utilization data; however, it is currently not used to capture patient-specific antimicrobial stewardship interventions. It is imperative to capture these interventions and analyze them to improve the program and patient outcomes.

Description: We developed an in-house database using Microsoft Access ${ }^{\circledR}$ to capture antimicrobial stewardship interventions at the patientspecific level. The database captures information under 3-categories: patient, visit, and intervention as shown below.

Action: For the image that goes with the "Action" section of this abstract, please see Abstract Appendix, available at https://www.cjhp-online.ca/ index.php/cjhp/issue/view/125/showToc

Evaluation: We evaluated 6-months of data from September 2016 February 2017. We were able to categorized types of interventions by frequency and identify which clinical care areas had the highest number of interventions. We found that the majority of IV-to-PO conversions were from piperacillin/tazobactam to amoxicillin-clavulanate. Meropenem to ertapenem was the leading IV-to-IV conversion. It took about 3-3.5 
days to convert from either IV-to-PO or IV-to-IV. Piperacillin/tazobactam was the leading antimicrobial for dose optimization. Finally, we were able to demonstrate saving of $\$ 15,000$ in direct antimicrobial costs during the first 6-months.

Implications: The database allows us to capture and analyze stewardship patient-specific interventions, which can be used to improve the program and share results with stakeholders. We can also utilize the database to calculate cost savings, acceptance rates, and benchmark physicians among peers.

\section{Performance of an Innovative Patient Decision Aid for Atrial Fibrillation Stroke Prevention Therapy}

Loewen $P^{l}$, Bansback $N^{1}$, Andrade ${ }^{1,2}$, Bonet $B^{4}$, Deyell $M^{1,3}$, Hicklin $J^{1}$, Kapanena $A^{1}$, Kwan $L^{4}$, Lynd L $L^{1}$, McLean $A^{1}, M c$ Gillivray $\mathcal{F}^{2}$, Salmasi $S^{1}$

${ }^{1}$ The University of British Columbia, Vancouver, BC

${ }^{2}$ Vancouver Coastal Health, Vancouver, BC

${ }^{3}$ Providence Health Care, Vancouver, BC

${ }^{4}$ Fraser Health, Vancouver, BC

Background: Choosing stroke prevention therapy in atrial fibrillation (SPAF) is complicated because it requires patients to make trade-offs and individual patient preferences are unpredictable. Misalignment between prescribed therapy and patient preferences is associated with poor therapy adherence and persistence and SPAF failure. To help address this misalignment, we have developed a unique online decision aid (DA), SPARC-DT, which integrates patients' stroke and bleeding risk, their values and preferences for key therapy attributes, and best evidence to identify which options are best matched with their preferences. Using SPARC-DT could be a basis for shared decision-making (SDM).

Objective: To evaluate the performance of SPARC-DT in patients with $\mathrm{AF}$ or at risk of $\mathrm{AF}$.

Methods: Design: Prospective observational study. Participants were AF patients or patients at risk of $\mathrm{AF}$ based on age $>50 \mathrm{y} / \mathrm{o}$ recruited from $\mathrm{AF}$ clinics and community centers. Consenting participants completed a pre-DA questionnaire, the SPARC-DT decision aid, and a post-DA questionnaire, either self-guided or by study personnel based on participant preference. Study outcomes were pre- vs. post-SPARC-DT AF knowledge assessment (AFKA), decisional conflict scale (DCS), System Usability Scale (SUS), and qualitative user feedback.

Results: 38 patients (mean age 71) completed the study. Using SPARCDT improved AFKA scores (7.93 vs. 8.61/10; $\mathrm{p}=0.02$ ), reduced overall decisional conflict (DCS $\Delta-21 / 100 ; \mathrm{p}<0.01$ ), and improved all DCS subscales (all $\mathrm{p}<0.05)$. Those "unsure" about their preferred therapy was reduced by $50 \%$. On the SUS and from qualitative feedback, most participants found SPARC-DT easy to use and learn, expressed willingness to use and confidence in using it, and found it to be well integrated.

Conclusion: SPARCT-DT performed well on all conventional measures of decision aid performance, reducing decisional conflict, increasing knowledge, and was deemed highly usable by participants. With refinement, SPARC-DT could be a valuable facilitator of SDM.

\section{Novel Student-Preceptor Models in Pharmacy Education: A Qualitative Analysis of the PharmD Student Experience}

McIntyre $C^{1,2}$, Natsheh $C^{1,2}$, Leblanc $K^{1,2}$, Fernandes $O^{1,2}$, Bjelajac-Mejia $A^{2}$, Raman-Wilms $L^{2}$, Cameron $K^{1,2}$

${ }^{1}$ University Health Network, Toronto, ON

${ }^{2}$ Leslie Dan Faculty of Pharmacy, University of Toronto, Toronto, ON

Background: Experiential rotation requirements in the Entry-to-Practice PharmD program have increased. To accommodate significantly more pharmacy learners on-site, institutions have explored novel studentpreceptor teaching models. These include peer-assisted-learning (PAL; 2 or more students of same educational level), near-peer-teaching (NPT; 1 or more junior students with 1 or more senior students), and copreceptorship (CoP; 2 or more preceptors).

Objective: To describe students' experiences and perceptions of novel teaching models and evaluate the effectiveness of these models on students' learning using Kirkpatrick's levels.

Methods: Pharmacy students from the class of 2015, 2016, and 2017 were invited to participate in semi-structure interviews. Interviews were transcribed, coded, and analyzed for themes. Themes were mapped according to the Kirkpatrick model for appraising educational training.

Results: Twenty semi-structured interviews were conducted with 10 pharmacy students from the class of 2017, and 5 each from the classes of 2016 and 2015. Forty-three experiences (19 CoP, 14 PAL, 10 NPT) were described from 14 institutions. Many themes overlapped between the three novel teaching models. In CoP, students described increased preceptor availability and exposure to different patient care approaches. Challenges arose when preceptors had different student expectations. Students overwhelmingly endorsed a multi-learner environment. Both PAL and NPT students felt well supported as collaboration with other learners was readily fostered. Potential challenges in PAL and NPT included student competitiveness and difficulties when personalities conflicted. All three models allowed for the development of skills including communication, collaboration, and teamwork. Because of their experiences, students reported an improvement in their approach to patient care. They also described a positive impact on their professional practice and approaches to teaching as new preceptors.

Conclusion: Pharmacy students enjoyed their experiences in novel student-preceptor models. These opportunities had a positive impact on overall learning during the rotations and as new practitioners.

\section{Evaluation of Physical Assessment Education for Practicing Pharmacists: A Cross-Sectional Study}

Barry $A^{1,2}$, Egan $G^{3}$, Turgeon $R^{4}$, Leung $M^{5}$

${ }^{1}$ Faculty of Pharmaceutical Sciences, University of British Columbia, Vancouver, $B C$

${ }^{2}$ Chilliwack General Hospital, Lower Mainland Pharmacy Services,

Chilliwack, $B C$

${ }^{3}$ Vancouver General Hospital, Lower Mainland Pharmacy Services,

Vancouver, $B C$

${ }^{4}$ Faculty of Medicine \& Dentistry (Division of Cardiology), University of Alberta, Edmonton, $A B$

${ }^{5}$ St. Paul's Hospital, Lower Mainland Pharmacy Services, Vancouver, BC

Background: Pharmacists are now seeking to incorporate physical assessment (PA) into their practice. This prompted the creation of a 30-hour PA course for practicing pharmacists developed by a Canadian Society of Hospital Pharmacists branch. 
Objectives: To evaluate pharmacists' knowledge, skills, and confidence with performing PA after completion of the course.

Methods: We invited all course participants via email to complete online surveys immediately and 6 months after completion of the course.

Results: Of 218 pharmacists who completed the course, 102 (46.8\%) completed the immediate post-course survey. Two-thirds of respondents did not use PA in their practice prior to the course. A lack of formal training and comfort were the most frequently selected barriers to performing PA prior to the course. All participants agreed the course improved their knowledge of PA and 96\% agreed it improved their skills. Most respondents (90\%) agreed the course improved their ability to care for patients. After the course, $60 \%$ and $67 \%$, respectively, agreed they felt confident performing PA or intervening on a patient's drug therapy based on their PA findings. The most common suggestion for course improvement was more hands-on practice. Twenty-five of 158 eligible participants (15.8\%) completed the 6-month post-course survey, of which $79 \%$ had performed PA in practice. Only $60 \%$ agreed they felt confident performing PA, whereas $84 \%$ now agreed they would intervene on a patient's drug therapy based on their PA findings. The most frequently selected barrier to performing PA was now lack of time. The primary limitation of this study was potential responder bias.

Conclusions: The course improved pharmacists' knowledge and skills with performing PA. Six months after the course, most respondents used PA in practice. While the proportion of participants that felt confident performing PA had not increased, more were willing to use their PA findings to change drug therapy.

\section{International Normalized Ratio Point of Care Testing in an Outpatient Anticoagulation Clinic and the Impact on the Patient Experience: A Quality Improvement Study}

Chisholm K, Culley C, Spina S

Department of Pharmacy, Island Health, Victoria, BC

Faculty of Pharmaceutical Sciences, University of British Columbia, Vancouver, $B C$

Background: Patients who are receiving warfarin therapy require ongoing blood testing to monitor their international normalized ratio (INR). In Canada, the standard for INR testing is by laboratory venipuncture. Pointof-care testing (POCT) is an alternative method, and previous studies have shown that it may enhance patient convenience and comfort. However, there is little information on the impact of INR POCT on the patient experience in a pharmacist-led, outpatient anticoagulation clinic.

Objective: To evaluate how the implementation of an INR POCT device for the initiation and stabilization of warfarin therapy in a pharmacist-led, outpatient anticoagulation clinic impacts the patient experience compared to laboratory testing.

Methods: We performed a prospective, before-and-after, quality improvement study of patients attending an outpatient anticoagulation clinic in Victoria BC, Canada. Using an anonymous patient survey, we evaluated outcomes measured before and after the implementation of an INR POCT device. Outcomes included patient time spent and number of visits to the hospital for anticoagulation-related care in one day, patient satisfaction with their blood testing experience, cost to patient, missed activities, and pain and discomfort. A one-month cost analysis was completed to compare health care cost of INR POCT and laboratory testing.
Results: Fifty-eight patients completed the survey. Patients undergoing POCT spent less time receiving anticoagulation-related care compared to laboratory testing (59.7 $\mathrm{min}$ vs $144.5 \mathrm{~min}$; $\mathrm{p}<0.05$ ). Using a 10 -point scale to quantify satisfaction with their blood testing experience, patients expressed higher satisfaction with POCT (9.6 vs. 7.8; $\mathrm{p}=0.001$ ). There were no differences in number of visits to the hospital, cost to patient, missed activities, or patient pain and discomfort. Health care cost was similar for both testing strategies when comparing one month of testing. Conclusions: Our findings support the continued use of INR POCT for the initiation and stabilization of warfarin therapy in a pharmacist-led, outpatient anticoagulation clinic.

\section{Case Report: Fatal Arrhythmia in a Patient Receiving an Aconite Herbal Product}

Lee J, Bucci C

Sunnybrook Health Sciences Centre, Toronto, ON

Background: Aconite is commonly used in traditional Chinese medicine. Aconite poisoning can lead to serious cardiac toxicity such as ventricular arrhythmias.

Case Description: A 44 year old male was found unconscious for an unknown duration. His rhythm showed ventricular tachycardia and fibrillation, requiring defibrillation and an amiodarone infusion. Arrhythmias subsided after 24 hours, however brain imaging was consistent with brain death. The family noted that he had ingested a large quantity of an herbal medication "Aconite". The patient had previously been boiling the medication prior to ingestion, but on the morning of presentation, he consumed a large handful without boiling.

Assessment of Causality: Based on the Naranjo scale, there is a "probable" chance of an adverse drug reaction (total score 6):

There are previous reports on this reaction $(+1)$

The event appeared after administration $(+2)$

There are no alternative causes that could have on their own caused the reaction $(+2)$

The reaction was confirmed by objective evidence $(+1)$

Literature Review: Aconite poisoning has been well documented in the Chinese literature. Patients may present with gastrointestinal (nausea and vomiting), neurologic (paresthesias), and cardiac symptoms (arrhythmias). Cardiac toxicity is thought to be related to the binding of fast voltage gated sodium channels, resulting in persistent depolarization. Processing (boiling) is recommended to hydrolyze the contents into less toxic derivatives. Amiodarone and flecainide have been used with some success, but no single agent have been shown to be of consistent benefit.

Importance to Practitioners: This case report is unique as it describes a fatal arrhythmia after ingestion of an herbal medication. With the increasing use of herbal products and the prevalence of Chinese immigrants in Canada, pharmacists can play an important role in identifying and assessing their potential harmful effects. 


\section{A Survey of the Use of Cannabis in Children at a Tertiary Teaching Hospital}

Moreno M, Vaillancourt R, Pouliot A, Sell E, Hevenor B, Viracoumarane K Children's Hospital of Eastern Ontario (CHEO), Ottawa, ON

Background: There has recently been an increase use of cannabis for medical purposes in the pediatric population. However, limited data on the efficacy and safety of cannabis in children has been published. A comprehensive characterization of cannabis use in this population can further contribute to a better understanding under which circumstances cannabis is being used and the observed outcomes.

Objectives: To perform a retrospective drug use evaluation to describe all inpatient and outpatient medical uses of cannabis in a tertiary teaching hospital over the last 3 years.

Methods: A retrospective medical record review was completed of inpatients and outpatients $<18$ years of age prescribed medical cannabis between May 1st, 2014 and May 1st, 2017. Patients using cannabis recreationally were excluded.

Results: There were a total of 300 unique patients identified and 37 children were included in this study. Of these, 30 patients were using medical cannabis and most were using it for seizures (93\%) where $82 \%$ were described as having intractable/ refractory seizures. Among these, $78 \%$ reported a decrease in seizures and of those $76 \%$ had a transient effect where a decrease was noted for 130.38 days $( \pm 99.14)$, but then seizures increased once again. Additionally, a subgroup of 7 patients were selfmedicating with cannabis. They obtained cannabis without proper authorization as a dried flower and were using it for chronic pain $(71 \%)$ and/or anxiety (71\%).

Conclusions: Cannabis use is reported more often for childhood refractory epilepsy compared to other chronic conditions. The use of cannabis in children with medication-refractory epilepsy may be warranted after trying first line anticonvulsant medications and after discussing non-pharmacological options. Based on our data, decrease in seizures may only be transient.

\section{Current Identification and Management Practices of Steroid-Induced Hyperglycemia in Brain and Spinal Tumour Patients on a Neurosurgical Unit}

Rankin $S^{1}$, Gilmour ${ }^{2}$, Halapy $H^{1,2}$, Wong $S^{2}$, Saccucci $P^{2}, N_{g} R^{2}$, Chan WWY ${ }^{1}$ University of Toronto, Toronto, $\mathrm{ON}$

${ }^{2}$ St. Michael's Hospital, Toronto, ON

Background: Brain and spinal tumor (BST) patients often receive dexamethasone to alleviate neurological symptoms. Steroid-induced hyperglycemia $(\mathrm{SIH})$ has been reported in $5-72 \%$ of Brain tumour patients. Hyperglycemia has been associated with worsened clinical outcomes, including shortened survival in brain tumour patients. We hypothesize that a gap exists in identifying and managing SIH in BST patients at our institution.

Objectives: We aim to describe the current identification and management practices of SIH in BST patients at our institution.

Methods: A retrospective chart review of adult BST patients admitted to the neurosurgical unit at a tertiary care hospital between January and December 2016, and received dexamethasone as well as diabetic medication(s), was performed. The primary endpoints included the average proportion of blood glucose $(\mathrm{BG})$ readings above $10 \mathrm{mmol} / \mathrm{L}$ per admission and the percentage of patients with: 1) BG monitoring for 48 hours after dexamethasone start; 2) incidences of hypoglycemia;
3) a start on corrective insulin scale for initial glycemic control; 4) an appropriate corrective insulin scale; 5) "STAT" dose of insulin; 6) change(s) to diabetic medication(s) at discharge; and 7) endocrine or medicine consult

Results: Thirty-six patients over 41 admissions were reviewed. The primary endpoints were: $61.7 \%$ was the average proportion of BG readings above $10 \mathrm{mmol} / \mathrm{L}, 92.7 \%$ of patients had BG monitoring for 48 hours after dexamethasone start, 4 incidences of hypoglycemia, $56 \%$ started on corrective insulin scale for initial glycemic control, $43.9 \%$ received appropriate corrective insulin scale, $26.8 \%$ received "STAT" dose insulin, $74 \%$ had change(s) to diabetic medication(s) at discharge, and $36.5 \%$ had endocrine or medicine consult.

Conclusion: While screening for SIH seems reasonable, the management of SIH at our institution appears to be sub-optimal and highly variable. These results will enable us to design a quality improvement project that optimizes SIH identification and management in BST patients.

\section{Clonidine for Sedation in Critically III Adults: A Retrospective Chart Review}

Purivatra $E^{1}$, Guenette $M^{1}$, Coleman $B^{3,4}$, Burry $L^{1,2}$

${ }^{1}$ Leslie Dan Faculty of Pharmacy, University of Toronto, Toronto, ON

${ }^{2}$ Department of Pharmacy, Mount Sinai Hospital, Toronto, ON

${ }^{3}$ Infectious Disease Research, Mount Sinai Hospital, Toronto, ON

${ }^{4}$ Dalla Lana School of Public Health, University of Toronto, Toronto, ON

Background: The alpha2 agonist clonidine may be used as an adjunct for Intensive Care Unit (ICU) sedation and analgesia to decrease traditional sedative and opioid requirements. However, use has been associated with hypotension and bradycardia.

Objectives: 1) To describe clonidine dosing regimens used for sedation in a mixed medical surgical ICU, as well as adverse events associated with use (i.e., hypotension, bradycardia, rebound withdrawal), and 2) to determine if clonidine has sparing effects on traditional drugs used for pain, sedation and agitation.

Methods: We conducted a retrospective chart review of all critically ill adult patients admitted who had received at least one dose of clonidine for sedation during a five-year study period. Patients were categorized for the analysis into low dose ( $\mathrm{LD} \leq 0.4 \mathrm{mg} /$ day) versus high dose $(\mathrm{HD}>0.4$ $\mathrm{mg} /$ day) based on the maximum total daily clonidine dose. Data was analyzed using the Mann Whitney $U$ test and Chi squared test for continuous and binary variables, respectively. A p value $<0.05$ was considered statistically significant.

Results: Of the 166 patients that met inclusion criteria, seventy-eight patients (47\%) had clonidine titrated to HD. There were no statistically significant differences in hypotension, bradycardia or withdrawal symptoms between the LD and HD clonidine groups. There was a greater reduction in mean daily opioid dose for HD clonidine versus LD (mean $-218.8 \mathrm{mcg}$ vs. $-42.5 \mathrm{mcg}$ of fentanyl equivalents, $\mathrm{p}=0.049$ ). The decrease in sedative usage post-clonidine initiation was not significant. Antipsychotic doses increased for HD compared to $\mathrm{LD}(5.7 \mathrm{mg}$ olanzapine equivalents vs $0 \mathrm{mg}, \mathrm{p}=0.04$ ).

Conclusions: Clonidine doses titrated beyond $0.4 \mathrm{mg} /$ day may decrease patients' opioid but not sedative requirements without causing statistically significant adverse effects. Antipsychotic doses increased as clonidine was titrated. 


\section{The Effect of Preoperative Cannabis Use on Opioid Consumption Following Surgery: A Cohort Analysis}

Jamal $N^{1}$, Korman $J^{1}$, Musing $M^{1}$, Malavade $A^{2}$, Coleman $B L^{3}$, Siddiqui $N^{2}$, Friedman $Z^{2}$

${ }^{1}$ Department of Pharmacy, Sinai Health System, University of Toronto, Toronto, $O N$

${ }^{2}$ Department of Anesthesia and Pain Management, Sinai Health System, University of Toronto, Toronto, $O N$

${ }^{3}$ Infectious Diseases Epidemiology Research Unit, Sinai Health System, University of Toronto, Toronto, $O N$

Background: Anecdotally, healthcare professionals on the Acute Pain Service have observed that individuals consuming cannabis preoperatively had higher opioid requirements in the postoperative period.

Objective: This study examined whether preoperative cannabis use impacted opioid consumption in the first 24 postoperative hours, in individuals undergoing abdominal surgery for inflammatory bowel disease (IBD).

Methods: We conducted a chart review of patients who underwent surgery for IBD at a tertiary, university affiliated hospital between January 2014 and December 2015. Patients were excluded if they used methadone preoperatively, used synthetic forms of delta-9-tetrahydrocannabinol preoperatively or received neuraxial analgesia. Demographic data, cannabis use, as well as data on perioperative analgesia was collected. Linear regression analysis was used to correct for potential confounding factors in order to compare the opioid consumption in the first 24 hours postoperatively between cannabis users (C) and non-users (NC).

Results: Of the 354 charts included, 42 (11.9\%) were classified in the C group and $312(88.1 \%)$ in the $\mathrm{NC}$ group. The $\mathrm{C}$ group was significantly $(\mathrm{p}<0.05)$ younger, with a higher percentage of males but did not differ with respect to type of surgery, length of surgery, preoperative opioid use or intraoperative opioid use compared with the NC group. Linear regression analysis demonstrated an increase in postoperative opioid consumption proportional to the amount of cannabis consumed preoperatively. The cannabis group required an additional $1.13 \mathrm{mg}$ (95\% CI: 1.02-1.25) morphine equivalent per gram of daily cannabis used $(\mathrm{p}=0.015)$.

Conclusions: Our results demonstrated that cannabis use increased the postoperative opioid consumption in patients undergoing IBD surgery. This could influence postoperative pain management and increase the risk of opioid side effects. Future prospective studies should expand beyond the IBD population and look at the role of synthetic cannabinoids as adjuncts for pain management.

\section{Stability of Extemporaneously-Compounded Temozolomide $10 \mathrm{mg} / \mathrm{mL}$ Suspension in Oral Mix SF in Glass and Plastic Bottles and Plastic Syringes}

Lingertat-Walsh $K H^{1}$, Weilnau ${ }^{\beta}$, Dupuis $L L^{1,6}$, Ostrenga $A^{4}$, Cober $M P^{3,5}$, Law $S^{2}$, Walker $S E^{2,6}$

${ }^{1}$ Departments of Pharmacy, Hospital for Sick Children, Toronto, ON

${ }^{2}$ Sunnybrook Health Sciences Centre, Toronto ON

${ }^{3}$ Akron Children's Hospital, Akron, $\mathrm{OH}$

${ }^{4}$ University of Mississippi Medical Center, Jackson, MS

${ }^{5}$ Northeast Ohio Medical University, College of Pharmacy, Rootstown, $\mathrm{OH}$

${ }^{6}$ Faculty of Pharmacy, University of Toronto, Toronto, $O N$

Background: Temozolomide oral suspension is not commercially available. Colour changes and crystal formation have been observed with previously published extemporaneous temozolomide suspension formulations.
Objective: To evaluate the stability of 3 temozolomide $10 \mathrm{mg} / \mathrm{mL}$ suspensions prepared in Oral Mix SF stored in 3 types of containers (amber glass, amber polyethylene terephthalate (PET) and polypropylene oral syringes) at $4^{\circ} \mathrm{C}$ and $25^{\circ} \mathrm{C}$.

Methods: Three separate batches of 3 different formulations in Oral Mix SF with (i) povidone K-30; (ii) povidone K-30 and citric acid and (iii) neither povidone $\mathrm{K}-30$ nor citric acid were made and were stored in 3 container types (amber glass, amber PET and oral syringes). Half of the aliquots in each container type was stored at $25^{\circ} \mathrm{C}$, the other half at $4{ }^{\circ} \mathrm{C}$, protected from light. On study days 0 , 5, 8, 14, 21, 28, 35, 42, 56, physical stability was assessed and the temozolomide concentration was determined using a stability-indicating method for samples drawn from each container type stored at each temperature. Chemical stability was based on the intersection of the lower limit of the $95 \%$ confidence interval (CI) of the observed degradation rate and the time to achieve $90 \%$ of the initial concentration (T-90[95\%]).

Results: The stability-indicating analytical method was accurate (deviation $<1.5 \%)$ and reproducible (CV\%<1.5\%). Samples stored at $25^{\circ} \mathrm{C}$ turned from white to orange. Temozolomide concentrations in all refrigerated samples in all container types remained above $90 \%$ of the initial concentration for 56-days, resulting in a T-90[95\%] exceeding 56-days. Analysis of variance demonstrated changes in concentration due to study day and temperature $(\mathrm{p}<0.001)$ but not container $(\mathrm{p}=0.991)$ or formulation $(\mathrm{p}=0.987)$.

Conclusions: Temozolomide $10 \mathrm{mg} / \mathrm{mL}$ oral suspension in Oral Mix SF with and without povidone K-30 or citric acid was chemically stable when stored at $4{ }^{\circ} \mathrm{C}$ and $25^{\circ} \mathrm{C}$ in in glass, PET, or oral syringes for 56 and 6-days, respectively.

\section{Stability of 20, 40 and $50 \mathrm{mcg} / \mathrm{mL}$ Fentanyl Solutions Stored in Syringes at Room Temperature $\left(23^{\circ} \mathrm{C}\right)$}

Riss $V^{1}$, Hook $R^{1}$, Low $A^{1}$, Scharrer $E^{1}$, Law $S^{2}$, Walker $S E^{2,3}$

${ }^{1}$ Departments of Pharmacy, Hospital for Sick Children, Toronto, ON

${ }^{2}$ Sunnybrook Health Sciences Centre, Toronto, ON

${ }^{3}$ Leslie Dan Faculty of Pharmacy, University of Toronto, Toronto, ON

Background: Inpatient hospital pharmacies must compound intravenous products and assign an appropriate beyond-use-date (BUD) as per NAPRA standards, because products are not commercially available. Furthermore, having medications in a Ready-To-Administer format on nursing units is important for safe and timely administration. Paediatric patients require lower concentrations of opioid continuous infusions than adults and while previous publications have demonstrated the stability of fentanyl, data for lower concentrations stored in syringes is not available.

Objective: We sought to evaluate the chemical stability fentanyl, prepared in syringes.

Methods: On study day 0, $50 \mathrm{~mL}$ solutions of $20 \mathrm{mcg} / \mathrm{mL}, 40 \mathrm{mcg} / \mathrm{mL}$ and $50 \mathrm{mcg} / \mathrm{mL}$ concentrations of fentanyl were prepared in BD syringes. 3 units of each container and concentration were stored at room temperature. Concentration analysis was completed on study days $0,1,3,7,14,24,38,57,77$ and 90 . Fentanyl concentrations were determined by a validated stability-indicating liquid chromatographic method with UV detection. Chemical stability was based on the intersection of the lower limit of the $95 \%$ confidence interval of the observed degradation rate and the time to achieve $90 \%$ of the initial concentration. 
Results: The analytical method separated degradation products from fentanyl such that the concentration was measured specifically, accurately (deviations from known averaged 2.5\%) and reproducibly (replicate error was less than $1.4 \%(\mathrm{CV}(\%))$. During the study period all solutions retained more than $96.7 \%$ of the initial concentration. Analysis of variance revealed significant differences in percent remaining due to concentration $(\mathrm{p}<0.001)$, and study day $(\mathrm{p}<0.001)$ although the study was capable of detecting less than a $0.51 \%$ difference in percent remaining. The calculated beyond-use-date was greater than 235days, exceeding the 90-day study period for all concentrations.

Conclusions: We conclude that 20 and $40 \mathrm{mcg} / \mathrm{mL}$ solutions of fentanyl diluted in saline and $50 \mathrm{mcg} / \mathrm{mL}$ undiluted solutions of fentanyl, stored in polypropylene $\mathrm{BD}$ syringes are physically and chemically stable for 90 days at room temperature $\left(23^{\circ} \mathrm{C}\right)$.

\section{Stability of Generic Formulations of Bortezomib 1.0 and $2.5 \mathrm{mg} / \mathrm{mL}$ in Vials and Syringes Stored $4^{\circ} \mathrm{C}$ and Room Temperature $\left(23^{\circ} \mathrm{C}\right)$}

Law $S^{1}$, Charbonneau LF', Iazzetta J', Perks W', Walker SE ${ }^{1,2}$

${ }^{1}$ Department of Pharmacy, Sunnybrook Health Sciences Centre, Toronto, ON ${ }^{2}$ Leslie Dan Faculty of Pharmacy, University of Toronto, Toronto, $O N$

Background: Generic versions of bortezomib raise questions about the reliability of extending stability study data from brand to brand.

Objective: To evaluate the stability of Janssen, Teva, Actavis and Dr.Reddy's bortezomib formulations reconstituted to produce either 1.0 or $2.5 \mathrm{mg} / \mathrm{mL}$, during storage over at least 21-days at room temperature $\left(23^{\circ} \mathrm{C}\right)$, and under refrigeration $\left(4^{\circ} \mathrm{C}\right)$ in plastic syringes and manufacturer vials.

Methods: On study day $0,2.5 \mathrm{mg} / \mathrm{mL}$ and $1.0 \mathrm{mg} / \mathrm{mL}$ concentrations of the Janssen, Teva, Actavis and Dr.Reddy's formulations were prepared. Three units of each container were stored at $23^{\circ} \mathrm{C}$ and 3 at $4^{\circ} \mathrm{C}$. Concentration and physical inspection were completed on at least 8-study days over a 21-42 day study period. Bortezomib concentrations were determined by a validated stability-indicating liquid chromatographic method with UV detection. The end point of these studies is the time to achieve $90 \%$ of the initial concentration (T-90). This was completed with confidence determining the intersection of the lower limit of the $95 \%$ confidence interval of the observed degradation rate and $90 \%$ of the initial concentration (T-90[95\%]).

Results: The analytical method separated degradation products from bortezomib during all studies such that the concentration was measured specifically, accurately (deviations less than $2.5 \%$ ) and reproducibly (replicate error averaged 2.5\%). During all studies, all solutions retained more than $94 \%$ of the initial concentration. The T-90[95\%] exceeded the study period for all formulations at all temperatures, concentrations and container combinations. Analysis of variance failed to detect significant differences between manufacturers ( $\mathrm{p}=0.970$ ).

Conclusions: We conclude that formulations of bortezomib currently marketed in Canada by Janssen, Teva, Actavis or Dr.Reddy's, reconstituted with $1.4 \mathrm{~mL}$ or $3.5 \mathrm{~mL}$ of $0.9 \%$ sodium chloride to create solutions containing $2.5 \mathrm{mg} / \mathrm{mL}$ or $1.0 \mathrm{mg} / \mathrm{mL}$, respectively, are physically and chemically stable for more than 21 -days at $4^{\circ} \mathrm{C}$ or $23^{\circ} \mathrm{C}$ in both syringes and the manufacturer's glass vial.

\section{Stability of 4 and $10 \mathrm{mcg} / \mathrm{mL}$ Remifentanil Solutions Stored in Syringes at Room Temperature $\left(23^{\circ} \mathrm{C}\right)$}

Hook $R^{1}$, Riss $V^{1}$, Scharrer $E^{1}$, Law $S^{2}$, Walker $S E^{2,3}$

${ }^{1}$ Departments of Pharmacy, Hospital for Sick Children, Toronto, ON

${ }^{2}$ Sunnybrook Health Sciences Centre, Toronto, ON

${ }^{3}$ Leslie Dan Faculty of Pharmacy, University of Toronto, Toronto, ON

Background: Inpatient hospital pharmacies must compound intravenous products and assign an appropriate beyond-use-date (BUD) as per NAPRA standards, because products are not commercially available. Furthermore, having medications in a Ready-To-Administer format on nursing units is important for safe and timely administration. Paediatric patients require lower concentrations of opioid continuous infusions than adults and while previous publications have demonstrated the stability of remifentanil, data for lower concentrations stored in syringes is not available.

Objective: We sought to evaluate the chemical stability remifentanil, prepared in syringes.

Methods: On study day 0, $5 \mathrm{~mL}$ solutions of $4 \mathrm{mcg} / \mathrm{mL}$ and $10 \mathrm{mcg} / \mathrm{mL}$ concentrations of remifentanil were prepared in $5 \mathrm{~mL}$ BD syringes. 3 units of each container and concentration were stored at room temperature. Concentration analysis was completed on study days $0,1,3,7,14,24$, $38,56,76$ and 90 . Remifentanil concentrations were determined by a validated stability-indicating liquid chromatographic method with UV detection. Chemical stability was based on the intersection of the lower limit of the $95 \%$ confidence interval of the observed degradation rate and the time to achieve $90 \%$ of the initial concentration (T-90).

Results: The analytical method separated degradation products from remifentanil such that the concentration was measured specifically, accurately (deviations from known averaged $2.65 \%$ ) and reproducibly (replicate error averaged $0.82 \%(\mathrm{CV}(\%))$. During the study period all solutions retained more than $96.96 \%$ of the initial concentration. Analysis of variance revealed significant differences in percent remaining due to study day $(p=0.002)$ but not concentration $(p=0.203)$. The study was capable of detecting a difference of more than a $0.76 \%$. The difference due to concentration was less than $0.5 \%$. The calculated T- 90 , with $95 \%$ confidence, exceeded 156 days for both concentrations.

Conclusions: We conclude that 4 and $10 \mathrm{mcg} / \mathrm{mL}$ solutions of remifentanil diluted in saline stored in polypropylene $\mathrm{BD}$ syringes are physically and chemically stable for 90 days at room temperature $\left(23^{\circ} \mathrm{C}\right)$.

\section{Satisfaction des établissements de santé suite à la mise en place d'une plateforme web de surveillance environnementale}

\section{Chauchat $L^{1}$, Bergeron $M^{1}$, Tanguay $C^{1}$, Bussières $J F^{1,2}$}

${ }^{1}$ Unité de recherche en pratique pharmaceutique, Département de pharmacie, Centre Hospitalier Universitaire Sainte-Justine, Montréal, QC

${ }^{2}$ Faculté de pharmacie, Université de Montréal, Montréal, QC

Contexte : Une étude annuelle de surveillance environnementale de traces de médicaments dangereux est menée au Canada depuis 2008 auprès des établissements de santé participant. Jusqu’à maintenant, la communication des rapports individualisés se faisaient par courriel.

Objectif : Évaluer la satisfaction des établissements de santé à la mise en place d'une plate-forme web contenant le rapport surveillance environnementale.

Méthodologie: Étude descriptive transversale. Une plate-forme web sur un serveur Linux (PHP) a été développée avec une base de données MySQL sur un domaine sécurisé par une connexion SSL (HTTPS). La plate-forme inclut les données actuelles et historiques de tous les établisse- 
ments et permet l'affichage et l'impression en temps réel des données d'un établissement comparées aux données agrégées de tous les établissements. La personne contact de chaque établissement a été invitée à répondre à un questionnaire en ligne (14 questions) visant à évaluer leur satisfaction.

Résultats : La plate-forme a été lancée le $1^{\text {er }}$ octobre 2017 auprès des 83 établissements ayant participé à l'étude 2017. Quarante-trois répondants (taux de participation de 51,8\%) ont complété le sondage. Quinze répondants (35\%) avaient plus de 15 civières en oncologie. Trente (70\%) ont participé à plus de deux études jusqu’à maintenant. Quarante-et-un (95\%) ont dit préféré avoir un accès en ligne en temps réel par rapport aux rapports papier, $43(100 \%)$ se sont dit satisfaits de la présentation visuelle des données et 38 (88\%) ont apprécié le format d'impression. Douze (28\%) ont rencontré des difficultés d'accès au départ compte tenu de la nécessité de recopier un hyperlien et de blocages liés à certains pare-feu. Ces problèmes ont été résolus.

Conclusion : Cette étude décrit le déploiement réussi d'une plateforme web d'accès aux rapports de surveillance environnementale. Cet accès facilité pourrait encourager le partage des données avec les équipes de chaque établissement.

\section{Medication Fluids in the Intensive Care Unit}

Ignacy $T^{1}$, Duffett $M^{1,2}$, Carlin $S^{1}$

${ }^{1}$ Hamilton Health Sciences, Hamilton, ON

${ }^{2}$ McMaster University, Hamilton, $O N$

Background: Fluid administration is essential to the care of critically ill patients; however fluid overload is associated with increased morbidity and mortality. Strategies to reduce fluid administration in this patient population must consider the contribution of medications, but this has not been well characterized.

Objectives: Our primary objective was to determine the contribution of medications to overall fluid administered in the intensive care unit (ICU). Secondary objectives include describing how fluid administration changes over time, and whether medication fluid volume can be reduced.

Methods: This retrospective observational study included patients admitted for $>24 \mathrm{~h}$ to either of two adult tertiary care ICUs between July 1 and October 31, 2016. We selected a random sample of 50 patients. We collected baseline demographics, daily fluid intake categorized by fluid type, and medication dose, concentration, and the associated fluid. We identified the maximum concentrations of medications using Micromedex, Pediatric Injectable Drugs and the Ottawa Manual.

Results: The median total volume of fluid administered is highest on day 1 at $3910 \mathrm{~mL} /$ day and decreases over time. Medications contribute $20 \%$ of fluids administered in the ICU. Throughout patients' stay in ICU, medication fluids maintain a proportional contribution of $20 \%$. Antimicrobials are the largest contributor of medication fluid. Among the top five antimicrobials used, medication fluid could be reduced by up to $71 \%$.

Conclusions: We identified opportunities to dramatically reduce the fluid from medications. Future studies should identify which patient populations would most benefit from limiting fluid volume. Any interventions to reduce fluid intake should include assessing the feasibility of further concentrating intravenous medications.

\section{Drug Use Evaluation of Oxycodone at a Canadian Teaching Hospital}

Wei $H^{1}$, Too $A^{1}$, Tanzini $R^{2}$, Satchu $S^{2}$, Dewhurst $N F^{1,2}$

${ }^{1}$ Leslie Dan Faculty of Pharmacy, University of Toronto, Toronto, ON ${ }^{2}$ St. Michael's Hospital, Toronto, ON

Background: Harms associated with prescription opioids are of increasing public concern. Oxycodone lacks superiority compared to other opioids, with an increased abuse potential. Since oxycodone's removal from the formulary at a Canadian hospital in 2012, its usage has not been characterized. We hypothesized that oxycodone was over-utilized and that opportunities exist for use optimization.

Objectives: This study was designed to characterize oxycodone use in concordance with evidence-based assessment criteria.

Methods: A retrospective observational drug use evaluation (DUE) was conducted for a four month period (April 1 to July 31, 2016) at a Canadian, urban, university-affiliated, tertiary care centre. Inpatients who received at least one dose of oxycodone immediate (IR) or controlled released (CR) were included. The primary measure of the evidence-based assessment criteria was the proportion of patients who received oxycodone $\mathrm{CR}$ in hospital, and were also stabilized on a CR formulation prior to admission. Patients were identified using the pharmacy computer system. Patients' electronic charts were reviewed using a standardized data collection form. Descriptive statistical analyses were performed.

Results: A total of 147 patient encounters with 248 orders for oxycodone were identified. Of oxycodone CR orders, 74/91 (81\%) were deemed appropriate as per the primary measure. Most oxycodone (IR and CR) orders $(52 / 248$ [21\%]) were initiated on the orthopedic ward. Four of the five top oxycodone prescribers were acute pain service physicians. Patient controlled analgesia (PCA) use was observed in 46\% (67/147) of all patient encounters. Of the patients prescribed oxycodone $\mathrm{CR}$ on discharge, the majority (17/20 [85\%]) were taking a CR formulation prior to admission.

Conclusions: The majority of oxycodone utilized at our institution was deemed appropriate according to the DUE assessment criteria. Opportunities to further optimize its use may be explored through order set modification or prescriber education.

\section{Inpatient Insulin Stewardship Program: A Baseline Needs Assessment}

Halapy $H^{1,2}$, Bruno $B^{3}$, Chen $L^{1}$, Lubchansky $S^{3}, Y u C^{1,3}$

${ }^{1}$ Diabetes and Endocrinology Centre, St. Michael's Hospital, Toronto, ON

${ }^{2}$ Leslie Dan Faculty of Pharmacy, University of Toronto, Toronto, ON

${ }^{3}$ Department of Medicine, University of Toronto, Toronto, $\mathrm{ON}$

Background: Inpatient insulin use often occurs in a non-standardized way, including use of sliding scales without basal-bolus treatment as recommended by Diabetes Canada Clinical Practice Guidelines. These practices lead to poor glycemic control, which is associated with increased infection and prolonged hospitalization. A previous study at our institution indicated that $35 \%$ of inpatients have hyperglycemia, suggesting a need for improvement.

Objective: With the overall goal of improving inpatient diabetes management, a baseline hospital-wide needs assessment regarding diabetes management was conducted in order to identify key barriers regarding inpatient diabetes management to inform the development of an insulin stewardship program, with the use of tailored interventions. 
Methods: Surveys (online, hard-copy) and focus groups were conducted with medical trainees (residents, students), nurse practitioners, medical and surgical nurses, and clinical pharmacists. Surveys were analyzed using descriptive statistics. Focus group recordings were transcribed and analyzed thematically.

Results: Surveys ( $\mathrm{n}=84$ ) and focus groups $(\mathrm{n}=8)$ revealed provider-, patient- and systems-related barriers to inpatient diabetes management. Provider-related barriers included: 1) inconsistent insulin start education; 2) peri-operative insulin use; 3) hypoglycemia avoidance/ management; 4) converting between insulin regimens; 5) correctional insulin use; 6) blood glucose monitoring education; and 7) when to consult the endocrine service for diabetes management. Patient-related barriers included appropriate use of home insulin in the inpatient setting. System-related barriers included: 1) lack of knowledge and awareness of standardized insulin order sets; 2) lack of resources available to support diabetes management; and 3) lack of decision support for appropriate insulin ordering.

Conclusion: Based on survey and focus group data, an insulin stewardship program will be developed to target the above barriers. Interventions include revised insulin order sets coupled with decision support; development of cheat sheets, standardized care protocols and manuals; revision of professional roles (e.g. pharmacisits), and insulin ordering/ inpatient diabetes management education.

\section{Long-Term Beta-Blockers after Myocardial Infarction in the Contemporary Era: A Systematic Review}

Hong $J^{1}$, Barry $A^{1,2}$

${ }^{1}$ Faculty of Pharmaceutical Sciences, University of British Columbia, Vancouver, $B C$

${ }^{2}$ Chilliwack General Hospital, Lower Mainland Pharmacy Services, Chilliwack, $B C$

Background: Beta-blockers are currently recommended as standard of care for patients who experience a myocardial infarction (MI) primarily based on evidence from the pre-reperfusion era. The effect of longterm beta-blocker therapy in patients post-MI without left ventricular dysfunction in the modern era is unknown.

Objective: To review the evidence for long-term ( $\geq 1$ year) beta-blockers as secondary cardiovascular preventive therapy on all-cause mortality and major adverse cardiovascular events (MACE), specifically cardiovascular mortality, nonfatal MI, and nonfatal stroke.

Methods: A systematic search of EMBASE, MEDLINE, and CENTRAL (to September 2017) for randomized controlled trials or propensitymatched cohort studies published within the last 10 years that compared beta-blockers to no beta-blockers on discharge in patients post-MI without heart failure or reduced left ventricular ejection fraction $(<30 \%)$ was performed. Both authors independently completed the literature search, data extraction, and study analysis.

Results: Eight cohort studies were included. Median study population was 1838 and duration of beta-blocker use ranged from 1-5 years. Definition of left ventricular dysfunction and reperfusion strategies varied among studies. Two smaller studies showed a significant reduction in all-cause mortality, whereas no difference was observed in 5 studies. One study showed significantly reduced cardiovascular mortality at 1 year, but no difference at 5 years, and no difference in all-cause mortality at 1 and 5 years. None of the 4 studies that reported MACE showed a significant reduction with beta-blocker therapy. These data are limited by inherent limitations of observational studies.
Conclusions: Though heterogeneous, the majority of contemporary studies identified did not demonstrate a reduction in death or MACE with chronic beta-blocker therapy in patients post-MI without left ventricular dysfunction. In the absence of an appropriately designed randomized controlled trial, these data impart uncertainty regarding the current standard of practice. Consequently, beta-blocker therapy should be reassessed on a case-by-case basis in patients post-MI.

\section{Clinical Guide for Pharmacists to Evaluate Risks and Manage QTc Prolongation Drug-Drug Interactions}

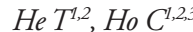

${ }^{1}$ Institute for Safe Medication Practices Canada, Toronto, ON

${ }^{2}$ School of Pharmacy, University of Waterloo, Waterloo, ON

${ }^{3}$ Leslie Dan Faculty of Pharmacy, University of Toronto, Toronto, ON

Background: There is an increasing number of medications with potential QTc prolongation risks and the subsequent degeneration into torsades de pointes $(\mathrm{TdP})$, a devastatingly fatal ventricular tachyarrhythmia.

Description: Our primary objectives were to identify recommendations posed by clinicians in evaluating QTc prolongation risks and to create a clinical algorithm or thought process aimed to help pharmacists in assessing and managing these drug-drug interactions.

Action: We focused on three commonly prescribed medications that were known to be associated with QTc prolongation risks (citalopram, domperidone, and ciprofloxacin). We conducted an environmental scan of recommendations made by national regulatory bodies and clinical guidelines; and performed a systematic review of primary literature between 2006 and 2016, with a primary focus on randomized controlled trials, systematic reviews, and meta-analyses.

Evaluation: We reviewed 7 articles. The primary literature, current recommendations from national regulatory authorities, and clinical guidelines consensually state that the evaluation of QTc prolongation risks requires a risk-benefit analysis of the drug combination. This analysis should be based on the severity of the drug-drug interactions, the patient's modifiable risk factors, and the mechanism in which the drug interaction results. In cases where the necessary doses may exceed the maximum dosing recommendations, pharmacists should ensure that baseline and steady-state 12-lead electrocardiograms are performed. Patients should be made aware of the signs and symptoms of abnormal arrhythmias and precipitating factors that may result in TdP. We developed a clinical algorithm to guide pharmacists in assessing and managing drug-drug interactions that involve potential QTc prolongation risks associated with these 3 medications.

Implications: Until further large-scale risk assessment tests and scoring can be performed, our clinical algorithm derived from a comprehensive environmental scan and literature review suggests that pharmacists should utilize their medication therapy expertise and effectively communicate potential risks of QTc prolongation to patients. 


\section{Trickle-Down Antimicrobial Stewardship: Reduction in Long-Term Care Resistance Rates Following Implementation of a Prospective Audit and Feedback Intervention in the Adjacent Acute Care Hospital}

\author{
Peragine $C^{1,2}$, Serbanescu $C^{2}$, Leis $J A^{3,4,5,6,7}$, Walker $S A N^{1,2,3,4 *}$ \\ ${ }^{1}$ Department of Pharmacy, Sunnybrook Health Sciences Centre, Toronto, ON \\ ${ }^{2}$ Leslie L. Dan Faculty of Pharmacy, University of Toronto, Toronto, ON \\ ${ }^{3}$ Division of Infectious Diseases, Sunnybrook Health Sciences Centre, Toronto, ON \\ ${ }^{4}$ Sunnybrook Health Sciences Centre Research Institute, Sunnybrook Health \\ Sciences Centre, Toronto, ON \\ ${ }^{5}$ Faculty of Medicine, University of Toronto, Toronto, $O N$ \\ ${ }^{6}$ Division of Infectious Diseases, Department of Medicine, Toronto, ON \\ ${ }^{7}$ Department of Infection Prevention and Control, Toronto, ON \\ * Senior Author; first-last-author-emphasis approach to authorship
}

Background: The Sunnybrook Health Sciences Center Bayview Campus is a shared site, home to a 627-bed acute care hospital and a 475-bed long-term-care (LTC) facility. A multidisciplinary antimicrobial stewardship (AMS) intervention was implemented in the acute care facility in October 2009. No specific intervention was initiated in LTC.

Objective: This study explores the impact of the acute care intervention on the burden of resistance in the adjoining LTC facility in the 7 years following the acute care program implementation.

Methods: Patient level data for clinical isolates of aerobic gram negative bacteria, Staphylococcus aureus, and Enterococcus spp. were obtained over a 14 year time period. Changes in the trend ( $\Delta$ slope) and level of resistance between the pre-intervention (October 2002 -September 2009) and post-intervention (October 2009 - September 2016) periods were assessed using interrupted time-series analyses with segmented regression. The primary outcome was the number of bacterial isolates exhibiting resistance to at least one therapeutically active antibiotic per month standardized to 10,000 patient days (PD) for all species collectively and all gram-negative species (GNs).

Results: A statistically significant reduction in resistance rate trend was found for all species collectively $(\Delta$ slope $=-0.97$ resistant isolates $/ 10,000$ $\mathrm{PD} /$ month, $\mathrm{p}=0.001)$ and all GNs $(\Delta$ slope $=-0.82$ resistant isolates/ $10,000 \mathrm{PD} /$ month, $\mathrm{p}=0.001)$. A significant reduction in the level of resistance was found for all bacteria beginning at post-intervention month $18(-3.24$ resistant isolates, $\mathrm{p}=0.023)$ and at post-intervention month 30 for all GNs ( -2.83 resistant isolates, $\mathrm{p}=0.046)$.

Conclusion: Time series modelling revealed that implementation of the acute care AMS program was associated with significant improvements in the adjoining LTC facility's antibiotic resistance rates, suggesting a trickle-down effect.

\section{Enhancing Mental Health Services through Hospital Outpatient Pharmacy and Assertive Community Treatment Team Collaboration}

\section{Davie S, Stutchbury R, Wist A}

Bluewater Health, Sarnia, ON

Background: The local Assertive Community Treatment (ACT) team is a multidisciplinary team working with chronic mental health patients in the community supported by the hospital. Outpatient community hospital pharmacy is ideal to resolve potential gaps in pharmaceutical care and further enhance services over traditional community pharmacy.

Description: Outpatient hospital pharmacy is able to provide individualized solutions for ACT team and their patients that will lead to outcomes that reduce workload, reduce amount of medication on site, improve storage conditions, increase clinical nursing time, implement educational initiatives and provide further support for both mental health inpatients and outpatients.

Action: The hospital outpatient pharmacy devised a business case to transition the medication distribution system for the local ACT team from a retail pharmacy outside the community in order to enhance workflow, increase medication monitoring, provide better transitional care and increase flexibility. The projected revenue enabled pharmacy to provide a full-time clinical pharmacist and pharmacy technician. Working closely with the ACT team, pharmacy was able to identify offsite ACT office clinical needs to ensure all necessary services, such as medication disposal, were improved to meet internal hospital standards.

Evaluation: Implementation increased nursing clinical availability and alleviated many technical aspects from their role. Pharmacy's involvement has been expanded to incorporate all facets of medication management to increase safety measures and reduce areas of risk, such as implementing improved storage and distribution solutions. Full-time access to a small clinical pharmacy team with the capacity for on call support has increased staff rapport and communication, reduced drug wastage, errors and improved transition of care in and out of hospital.

Implications: Outpatient pharmacy in a hospital setting is ideal to service hospital outpatient programs by providing the flexibility, availability, quality and understanding that a community pharmacy may not always be able to lend focus to.

\section{Vancomycin Every 4 hours in Paediatric Patients: A Case Series}

Gelinas $T^{1}$, Wong ETM ${ }^{1}$, Harris $V C^{1,2}$

${ }^{1}$ London Health Sciences Centre, London, ON

${ }^{2}$ Schulich School of Medicine \& Dentistry, Western University, London, ON

Background: Vancomycin target concentrations of $10-20 \mathrm{mg} / \mathrm{L}$ can be difficult to attain in paediatric patients due to their developmental pharmacokinetics. The usual starting dose for vancomycin is $15 \mathrm{mg} / \mathrm{kg}$ IV every 6 hours in infants and children. In some cases, pharmacists have used every 4 hour dosing in an attempt to reach therapeutic vancomycin concentrations.

Objectives: To review cases of hospitalized paediatric patients who have received vancomycin every 4 hours, in order to determine if this dosing interval appears safe and effective.

Methods: For this quality assurance case series, patients who had received vancomycin every 4 hours for $\geq 24$ hours were identified from a report of paediatric patients prescribed vancomycin from February 2014 until October 2017. Vancomycin trough levels, drawn within 30 minutes of the scheduled dose, were used as a surrogate marker for efficacy. Safety parameters included serum creatinine and urea, which were assessed at baseline and during vancomycin treatment. Descriptive statistics were used.

Results: A total of 18 paediatric patients, aged 6 months to 8 years old were included in the case series. All patients achieved therapeutic concentrations during their treatment. A total of 4 patients had supratherapeutic trough concentrations. Observations indicate that serum creatinine and urea trended upward as trough concentrations increased.

Conclusions: Our observations suggest that vancomycin dosing every 4 hours in the paediatric population correlates with a high frequency of supratherapeutic trough concentrations, along with increased urea and serum creatinine. Further investigation is required to identify which patients are most likely to benefit from every 4 hour vancomycin dosing and those more likely to experience supratherapeutic concentrations. 


\section{Comparison of Clinical Pharmacy Services in General Medicine and Surgery Patients: A Workload Measurement Study}

Peragine $C^{1,2}$, Walker $S E^{1,2}$

${ }^{1}$ Department of Pharmacy, Sunnybrook Health Sciences Centre, Toronto, ON ${ }^{2}$ Leslie L. Dan Faculty of Pharmacy, University of Toronto, Toronto, ON

Background: Canadian data describing the clinical services provided by hospital pharmacists (clinical pharmacy services, CPS) is lacking. Moreover, no Canadian study has examined if CPS demand and provision times differ for general medicine (GM) and surgery (GS) inpatients.

Objective: To describe the clinical services that hospital pharmacists provide, determine the incidence and provision times for various CPS, and identify differences in pharmacist workload as a function CPS and patient partition (GM or GS).

Methods: A data collection form to capture patient characteristics and CPS frequency and service times was developed. CPS activities included best possible medication history (BPMH), medication reconciliation, pharmacotherapy care plan, medication order review, insurance inquiries, dose clarification, therapeutic drug monitoring, antibiotic follow-up, thromboprophylaxis assessment, health care team discussions, inpatient counselling, discharge preparation activities, and "other" clinical activities. Ward pharmacists were asked to complete one documentation form for each patient admitted to their care over a 1 month period. Differences in patient characteristics, CPS frequency, CPS service times, total-timeper-patient, and daily-time-per-patient between GM and GS patients were evaluated using chi-squared and Mann-Whitney tests.

Results: Workload data for 273 GM patients and 191 GS patients were obtained. Inpatient counselling ( $8 \%$ vs. $3 \%, \mathrm{p}<0.05)$ and discharge preparation activities $(19 \%$ vs. $9 \%, \mathrm{p}<0.05)$ were reported more frequently for GM patients, while pharmacotherapy care plan (64\% vs. $77 \%$, $\mathrm{p}<0.05$ ), medication order review ( $7 \%$ vs. $29 \%, \mathrm{p}<0.05$ ), "other" activities ( $9 \%$ vs. $25 \%, \mathrm{p}<0.05$ ) were reported less frequently. Median service times for BPMH ( $15 \mathrm{~m}$ vs. $10 \mathrm{~m}, \mathrm{p}<0.001)$, pharmacotherapy care plan $(15 \mathrm{~min}$ vs. $10 \mathrm{~min}, \mathrm{p}<0.001)$, and "other" activities ( $10 \mathrm{~min}$ vs. $5 \mathrm{~min}, \mathrm{p}=0.019)$ were higher for GM patients. No difference in total-time-per-patient was detected. Daily-time-per-patient was higher for GS patients $(6 \mathrm{~min}$ vs. $4 \mathrm{~min}, \mathrm{p}<0.001$ ).

Conclusion: Significant differences in PCS frequency, PCS service times, and daily-time-per-patient were found between GM and GS patients.

\section{Validation of a Screening Tool to Assist in the Early Identification of Bloodstream Infection in Older Patients}

Walker $S A N^{1,2,3,4}$, Peragine $C^{1,2}, M a N^{1,2}$, Bannerman $H^{1}$, Elligsen $M^{1}$, Palmay $L^{1}$, Williams $E^{5,6}$, Liu $B^{6,7}$

${ }^{1}$ Department of Pharmacy, Sunnybrook Health Sciences Centre, Toronto, ON ${ }^{2}$ Leslie L. Dan Faculty of Pharmacy, University of Toronto, Toronto, ON

${ }^{3}$ Division of Infectious Diseases, Sunnybrook Health Sciences Centre, Toronto, ON ${ }^{4}$ Sunnybrook Health Sciences Centre Research Institute, Sunnybrook Health Sciences Centre, Toronto, ON

${ }^{5}$ Division Long-Term Care, Sunnybrook Health Sciences Centre, Toronto, ON

${ }^{6}$ Faculty of Medicine, University of Toronto, Toronto, $\mathrm{ON}$

${ }^{7}$ Division of Geriatric Medicine, Sunnybrook Health Sciences Centre, Toronto, ON

*Senior Author; sequence determines credit approach to authorship
Background: Delayed diagnosis of blood stream infection (BSI) occurs in $>20 \%$ of older patients. A validated BSI screening tool (ST) to identify older patients with a high probability of BSI may improve early diagnosis and management.

Objective: The objective was to validate a BSI-ST in older patients.

Methods: Inpatients $\geq 65$ years old admitted between March 12, 2010December 2, 2013 were eligible. A retrospective chart review of a matched cohort of older patients with and without documented BSI was completed. Data analysis was done for two age cohorts ( $\geq 80$ years old and 65-79 years old) and for all patients $\geq 65$ years old. Bacteremia pre-test probability, sensitivity, specificity, accuracy, false positive and negative rates of bacteremia, and positive and negative predictive value, post-test probability, and likelihood ratios were determined.

Results: The BSI-ST was validated in 310 patients. Of 25942 patients $\geq 65$ years old admitted during the study, 534 had BSI, corresponding to a period prevalence (PP) of BSI in older patients of $2.1 \%$. At the $2.1 \%$ BSI PP, the negative predictive value is $100 \%$ with a $0.4 \%$ probability of missing a BSI in an older patient.

Conclusion: The BSI-ST has excellent predictive capability for identifying older patients in whom a blood culture should be obtained, with a positive predictive value of $92 \%$ and false positive rate of only $10 \%$. Although the false negative rate was $20 \%$, a negative test in a patient with a BSI would occur in only $0.4 \%$ of patients at the institutional PP of BSI in older patients. Therefore, the retrospective validation of the BSI screening tool supports its implementation and pragmatic prospective evaluation.

For the table that goes with this abstract, please see Abstract Appendix, available at https://www.cjhp-online.ca/index.php/cjhp/issue/view/125/ showToc

\section{Patterns of Antimicrobial Use in an Outpatient Hemodialysis Unit}

Sivarajahkumar $S^{1,2}$, So $M^{1,2}$, Bell $C^{1,3,4}$, Morris $A^{1,3,4}$, Battistella $M^{1,2}$

${ }^{1}$ University Health Network, Toronto, $\mathrm{ON}$

${ }^{2}$ Leslie Dan Faculty of Pharmacy, University of Toronto, Toronto, ON

${ }^{3}$ Sinai Health System, Toronto, ON

${ }^{4}$ Faculty of Medicine, University of Toronto, Toronto, $\mathrm{ON}$

Background: Hemodialysis (HD) patients are at high risk for infections, including those caused by multi-drug resistant organisms (MDROs). Since antimicrobial exposure is the main risk factor for the emergence of MDROs, minimizing inappropriate antimicrobial use is imperative.

Objectives: To optimize antimicrobial use, it is first important to understand patterns of antimicrobial prescribing in the HD setting. The objectives of this study were to: (1) measure antimicrobial use, and (2) describe antimicrobial prescribing patterns, among patients receiving outpatient HD.

Methods: A retrospective observational cohort study was performed in an outpatient HD unit at an academic centre from February 1 - April 30, 2017. Eligible patients included adults who were prescribed at least one oral or intravenous (IV) antimicrobial by a hospital or community prescriber. Data were retrieved from the HD unit infection control database and analyzed using descriptive statistics. The primary outcome was total antimicrobial days of therapy (DOT) per 1000 patient-days. Secondary outcomes were antimicrobial prescriptions characterized by type of antimicrobial, purpose, indication, route, and prescriber type.

Results: A total of 53 patients were eligible for inclusion and 75 antimicrobial prescriptions were included for analysis. Antimicrobial use occurred in $53(16 \%)$ of 330 total outpatient HD patients. Over the 3-month study 
period, antimicrobial use was 27.5 DOTs/1000 patient-days. The most common indication for antimicrobial therapy was skin and soft tissue infection, followed by bloodstream infection and respiratory tract infection. Fluoroquinolones were the most frequently prescribed antimicrobials, accounting for $23 \%$ of prescriptions, while the second most prescribed were first-generation cephalosporins $(21 \%)$.

Conclusion: Overall, this study indicates that antimicrobial use was common, with 1 in $6 \mathrm{HD}$ patients receiving antimicrobials during the 3 -month study period. This may be the first study to describe prescribing patterns for both oral and IV antimicrobials in an outpatient HD population.

\section{Impact of an Antimicrobial Stewardship Bloodstream Infection Surveillance Program in Hospitalized Patients}

Dow $G^{1}$, MacLagran $T^{1}$, Allard ${ }^{2}$

${ }^{1}$ The Moncton Hospital, Horizon Health Network, Moncton, NB

${ }^{2}$ Universite de Moncton, Moncton, $N B$

Background: Bloodstream infections (BSI) in hospitalized patients represent sentinel events characterized by increased mortality. These infections represent an attractive stewardship opportunity because they warrant rapid initiation of empiric antimicrobial therapy, deft transition to directed (gram stain guided) and definitive (susceptibility guided) therapy.

Objectives: To measure the impact of a bloodstream infection surveillance program (BISP) on the adequacy and timing of antimicrobial therapy in hospitalized patients and model factors impacting antimicrobial cost, length of stay, and mortality in BSI patients.

Methods: A pre-post study design by retrospective chart review was carried out 18 months before and 18 months after initiation of a hospital BISP. The hospital ward and attending physician were notified of all positive blood cultures pre-intervention. Post-intervention an infectious disease pharmacist collaborating with an infectious disease consultant was notified in addition to standard notifications. Pre- and post-groups were compared using t-tests and Fisher exact tests. Antimicrobial costs and mortality were analysed using log-linear and logistic models, respectively.

Results: BSI were identified in 226 patients pre-intervention and 195 patients post-intervention. The two cohorts were similar in baseline characteristics and source of infection. E. coli, S. aureus, other enterobacteriaceae and beta-hemolytic streptococci were the most common bloodstream isolates. The post-intervention cohort received directed therapy 4.36 hours earlier $(\mathrm{p}=0.003)$, were more

\section{Epidemiology of Carbapenemase-Producing Enterobacteriaceae Bacteremia and Evaluation of Antimicrobial Prescribing Practices in a Community Hospital Setting}

Pang $S^{1}$, Haghshenas $E^{1}$, Richardson $D^{1}$, Baqi $M^{2}$

${ }^{1}$ William Osler Health System: Brampton Civic Hospital, Brampton, ON

${ }^{2}$ William Osler Health System: Etobicoke General Hospital, Etobicoke, ON

Background: The increasing incidence of carbapenemase-producing Enterobacteriaceae (CPE) bacteremia is a growing concern. With mortality rates exceeding $50 \%$, there remains no consensus surrounding optimal antimicrobial treatment. There is little literature pertaining to $\mathrm{CPE}$ in Canada. The majority of isolates submitted to our provincial Public Health Laboratory are from our health system's service area.
Objectives: This study aims to characterize our health system's CPE bacteremia population and evaluate the antimicrobials used for treatment, including the effect of monotherapy versus combination therapy on mortality.

Methods: A dual-centre retrospective chart review was conducted of adult CPE bacteremia patients admitted between January 1, 2010 and April 30, 2017. Baseline demographics included out-of-country hospitalization, causative organism, and susceptibilities. Measured outcomes included length of hospitalization, mortality within 30 days of index blood cultures, and re-admission within 30 days of discharge. Prescribing pattern data included antimicrobial agent, dosing regimen, and use of monotherapy or combination therapy.

Results: Thirteen cases of CPE bacteremia were reviewed. They most frequently occurred in patients with prior hospitalization outside of Canada, particularly in the Indian subcontinent (9/13, 69\%). Ninety-two percent (12/13) of isolates produced New Delhi metallo-beta-lactamase. All isolates were susceptible or intermediate to tigecycline. Tigecyline and colistin were the most commonly prescribed antimicrobials, respectively used in $62 \%(8 / 13)$ and $54 \%(7 / 13)$ of cases. Thirty-day mortality and readmission rates were $54 \%(7 / 13)$ and $50 \%(3 / 6)$, respectively. Survivors were 4 times more likely to have received combination therapy versus monotherapy (Odds ratio: 4.0, 95\% confidence interval: $0.36-41.11$, $P=0.26)$.

Conclusions: This study highlights the largest Canadian CPE bacteremia cohort to date. Our results suggest that combination therapy is more effective than monotherapy in improving survival outcomes. The inclusion of tigecycline as part of empiric combination therapy should be considered when CPE bacteremia is being considered or confirmed within our health system.

\section{Development and Implementation of a Provincial Beta-Lactam Allergy Management Initiative}

Landry $D^{1}$, MacLaggan $T^{2}$

${ }^{1}$ Dr. Georges-L.-Dumont University Hospital Centre, Vitalité Health

Network, Moncton, NB

${ }^{2}$ The Moncton Hospital, Horizon Health Network, Moncton, NB

Background: Beta-lactam allergies are often over diagnosed and over reported. Up to $10 \%$ of the population will report a penicillin allergy; however, as many as $95 \%$ of these patients are not truly allergic. Healthcare providers are more likely to prescribe non beta-lactam alternatives to these patients, which may be: less effective, more toxic, broader spectrum, more expensive and more likely to lead to infection or colonization with resistant organisms.

Description: The Provincial Anti-Infective Stewardship Committee (ASC) includes members from the two regional health authorities. Among its delegated tasks, the ASC develops educational resources and guidelines to improve the appropriateness, safety and cost-effectiveness of antiinfective use.

Action: The ASC developed the "Management of Penicillin and BetaLactam Allergy" guideline along with an executive summary document that condensed the main recommendations into a pocket sized booklet. A month-long educational and communication initiative was executed for their launch. The implementation initiative included announcement memos, educational bulletins, e-learning and live educational sessions. PRE/POST surveys were also sent to all health professionals of the target audience within both regional health authorities to assess the impact of the targeted educational program paired with the implementation of the guideline. 
Evaluation: Response rates for the PRE and POST surveys were low at $7.8 \%$ and $3.9 \%$ respectively. The table summarizes key results from the surveys.

Implications: Results showed that there was a significant degree of interest in the guidelines and a trend in improvement in antibiotic related knowledge and feelings of preparedness; however, response rates were poor and $54 \%$ felt their practice had not changed. Results highlight the importance of active interventions to engage practice change in antimicrobial stewardship.

For the table that goes with this abstract, please see Abstract Appendix, available at https://www.cjhp-online.ca/index.php/cjhp/issue/view/ $125 /$ showToc

\section{Factors Affecting Time to Fill Antiplatelet Therapy for Patients Discharged from Hospital}

\author{
Mulrooney $S^{1,2}$, Urquhart $E^{2}$, Harris $R^{2}$ \\ ${ }^{1}$ School of Pharmacy, Dalhousie University, Halifax, NS \\ ${ }^{2}$ Horizon Health Network, Saint John, NB
}

Background: Delays in filling antiplatelet therapy following hospital discharge have been identified in other parts of North America for patients who have undergone percutaneous coronary intervention (PCI) to treat acute coronary syndrome (ACS). These delays have been associated with an increased risk of recurrent myocardial infarction and all-cause mortality. It is unknown if delays exist for patients at the study site and what factors may affect this.

Objectives: The purpose of this study was to determine if delays exist for patients in the study area, and if so, the impact on hospital readmission rates. The secondary objective was to assess the impact of medication attitude, distance to home from site of discharge, site of discharge and medication insurance status on the time to fill antiplatelet therapy.

Methods: Prospective, observational, single-centre pilot project; conducted between January-April 2017. Patients 19 years of age and older, admitted to undergo PCI to treat ACS and discharged on clopidogrel, prasugrel or ticagrelor were eligible for enrolment during the index hospitalization. At 7-10 days post-discharge, prescription filling data was collected by telephone survey with participant and participant's community pharmacy. The Merck Adherence Estimator was administered at this time to gauge participant medication attitudes. Readmission to hospital data was collected from the provincial Electronic Health Record (EHR) 30-45 days post-discharge.

Results: Nineteen participants (79.2\%) reported filling a prescription for their antiplatelet agent on the day of discharge. No statistically significant delay was identified. Merck Adherence Estimator score ( $\mathrm{p}=0.0463$ ) and distance to home from site of discharge $(\mathrm{p}=0.0062)$ were identified as factors impacting time to fill.

Conclusions: Delays in filling antiplatelet therapy may not be an issue for our patients. This should be confirmed with further investigation. The Merck Adherence Estimator may be an appropriate tool to screen patients who may be at risk of experiencing delays.

\section{Evaluation of the Impact of Pharmacist-Led Penicillin Allergy Assessments on Antibiotic Utilization in a Large Community Teaching Hospital \\ Saleh $M^{1}$, Landry $C^{1,2}$, Do $K^{1}$, Khan $I^{1}$, Chagnon $N^{1,2}$, Chauret $D^{1,2}$ \\ ${ }^{1}$ Hopital Montfort, Ottawa, ON \\ ${ }^{2}$ Institut de Savoir Montfort, Ottawa, ON}

Background: Penicillins are the most common cause of allergic drug reactions with a prevalence of up to $20 \%$ in hospitalized patients. To date, there are limited Canadian publications describing pharmacist involvement in penicillin skin-testing. The purpose of this study is to evaluate the impact of a pharmacist-led initiative in a community hospital on de-labelling penicillin allergies and reducing the use of two broad spectrum antibiotics, meropenem and vancomycin.

Objectives: To determine the proportion of patients in whom an antibiotic change was made as a result of a penicillin allergy assessment and identify barriers for not de-escalating therapy in patients deemed non penicillin allergic. Potential drug cost savings were also examined for skin-tested patients.

Methods: This is an observational cohort study conducted in a community teaching hospital between October $1^{\text {st }} 2016$ and May $31^{\text {st }} 2017$, following the implementation of a policy allowing pharmacists to refer patients to an inpatient allergist for skin testing.

Results: Pharmacists recommended a penicillin skin test (PST) for 15 of 32 identified patients $(46.9 \%)$ with a penicillin allergy who were prescribed meropenem or vancomycin. Nine of 15 eligible patients $(60 \%)$ underwent a PST, with five patients having their antimicrobial therapy de-escalated to a beta-lactam antibiotic. Four patients had their therapy modified based only on the pharmacist assessment. De-escalation of therapy led to an average cost saving of was $\$ 137.89$ for patients switched to a beta-lactam after PST.

Conclusion: A minimal-cost, pharmacist-led initiative to reduce broadspectrum antibiotic use in penicillin allergic patients resulted in antimicrobial de-escalation in nine patients, demonstrating another opportunity for pharmacist involvement in antimicrobial stewardship.

\section{Pharmacy Technician Continuing Education Program at a Large Teaching Hospital}

Natsheh $C^{1,2}$, Cameron $K^{1,2}$

${ }^{1}$ University Health Network, Toronto, ON

${ }^{2}$ Leslie Dan Faculty of Pharmacy, University of Toronto, Toronto, ON

Background: Provincial College of Pharmacists mandates that its members maintain professional knowledge and skills appropriate for their scope of practice. At a large, teaching hospital, pharmacists have multiple opportunities to receive continuing education (CE) in an organized process; however, technician CE was not yet established.

Description: To describe the design and delivery of a structured, CE program for pharmacy technicians.

Action: A pilot was initiated in 2012 based on the structure of an existing pharmacist CE. It consisted of 30 minute, monthly presentations delivered at each site by pharmacy students. In 2013, an anonymous, needs assessment survey was sent for feedback. Comments were analyzed and incorporated into developing the final program launched in 2013.

Evaluation: Data from January 2014 to June 2017 was collected from 2 main sources; needs assessment surveys in 2013 and 2015 (total 33 respondents) and written feedback collected after presentations. Results showed that presentation topics were initially predominated by clinical therapeutic foci. However, over time, there was increase in the numbers of non-therapeutic topics reflecting real-time incorporation of technicians' 
feedback. There was equal interest for therapeutic and non-therapeutic topics. Pharmacy students comprised majority of presenters (90\%), pharmacists and technicians represented 5\% each. Forty-one percent of respondents expressed most interest in receiving education from peers (other technicians). Ninety percent felt more knowledgeable about the topic presented and $71 \%$ found the sessions were meeting their educational needs. Some common challenges to attending CE was workload, presentation time of day, location, and lack of interest in the topic.

Implications: A pharmacy technician CE program has been a sustainable initiative at our institution. A lead facilitator is necessary to coordinate the schedule and recruit presenters. Future directions include standardization of feedback survey to capture timely feedback, leverage digital media to increase access and explore opportunities to enable more peer-technician presenters.

\section{Questions to Ask about Your Medications}

\author{
Watt $A^{1}$, Sever $L^{1}$, Hyland $S^{1}$, Herold $D^{2}$, Hughes $L^{2}$, Murray $M^{2}$, Cass $M^{3}$ \\ ${ }^{I}$ Institute for Safe Medication Practices Canada, Toronto, ON \\ ${ }^{2}$ Patients for Patient Safety Canada, Edmonton, $A B$ \\ ${ }^{3}$ Canadian Patient Safety Institute, Edmonton, $A B$
}

Background: Patients are the constant in every transition of care and can be at high risk of adverse drug events, particularly at discharge from hospital to home. Knowing the right questions to ask can empower patients to be an active partner in their health and can help to mitigate risk of harm from medications.

Description: "5 Questions to Ask" is a national campaign to help patients and hospital pharmacists engage in a conversation about medication safety.

Action: The intervention was to develop, test, evaluate and disseminate a medication safety 'checklist' for use by patients and healthcare providers. Through small tests of change the 'checklist' was re-designed to the '5 Questions'.

Evaluation: A national online survey of patients and healthcare providers $(n=291)$ revealed that $85 \%$ of patients would feel comfortable asking their healthcare provider the ' 5 Questions', $84 \%$ of healthcare providers would be willing to answer their patient's ' 5 Questions' and 75\% of patients responded that the answers to these ' 5 Questions' would be very useful to help them understand their medications. A key performance measure was the extent of collaboration and use of the ' 5 Questions' among healthcare organizations. More than 150 Canadian organizations, at local, provincial and national levels have formally endorsed the '5 Questions' and implemented programs to increase reach and dissemination. Collective evaluation results demonstrate a commitment to a shared aim of empowering patients with questions to ask about their medications. There have been over 30,000 downloads of the poster since launch. The YouTube video has been viewed over 4000 times since its launch September 2016. Social media measures are also being collected.

Implications: The '5 Questions' with visible organization endorsements and translation in over 22 languages has demonstrated a shared interest in empowering patients. These questions may be a useful counselling framework or teach-back tool to help hospital pharmacists communicate more effectively with patients so they can better understand how to safely use their medications before they leave the hospital.

\section{A Pharmacy-Led Interdisciplinary Teaching Model in Specialized Pharmacotherapy: An HIV Pharmacy Rotation for Medical Residents.}

Naccarato $M^{1,2}$, Yoong $D^{1,2}$, Gough $K^{2,3}$, Arbess $G^{3,4}$

${ }^{1}$ Department of Pharmacy, St. Michael's Hospital, Toronto, ON

${ }^{2}$ Division of Infectious Diseases, St. Michael's Hospital, Toronto, ON

${ }^{3}$ Faculty of Medicine, University of Toronto, Toronto, $O N$

${ }^{4}$ Department of Family \& Community Medicine, St. Michael's Hospital, Toronto, $O N$

Background: The medical training model has traditionally been physician-to-physician, contrasting the current interdisciplinary healthcare practice environment. Pharmacists with specialized drug knowledge are well-positioned to provide expert training in complex antiretroviral therapy. We developed a collaborative teaching model, incorporating a pharmacist-led rotation for medical residents to learn specialized HIV pharmacotherapy.

Description: In 2010 a postgraduate residency program was inaugurated in which family practice medical residents could receive focused training in the delivery of HIV care. The provision of HIV care, however, is multifaceted and drug treatment can be complex. Thus, in 2013, a specialized HIV pharmacy rotation at our university-affiliated ambulatory clinic was created and offered to these family practice residents as an elective, with the primary goal to augment HIV drug knowledge and better equip physicians to providing exemplary HIV care. The concept was for the medical trainee to "become the pharmacist", learning to recognize and manage common drug-therapy issues in HIV-infected persons.

Action: A formalized curriculum was developed, including rotation goals to foster key competencies in family medicine while addressing gaps in pharmacology training and HIV care. The rotation could be tailored to the resident but generally consists of a core set of one-on-one teaching, case-based learning, and application of pharmacotherapeutics in a busy specialized ambulatory HIV clinic.

Evaluation: A pre- and post-rotation survey was developed to assess whether the pharmacy rotation improved the resident's confidence in their knowledge of HIV pharmacotherapy. This survey also gathered feedback and recommendations to enhance future rotations. Thus far, 5 medical residents have completed this residency with the elective pharmacy rotation and have strongly endorsed the rotation as valuable to their future practice.

Implications: This collaborative teaching model appears to be extremely valuable to enhance patient care and should be considered in other highlyspecialized pharmacotherapeutic areas.

\section{Influence of Manufacturer on Cefazolin Stability}

Xu $Y$, Walker SE

Leslie Dan Faculty of Pharmacy, University of Toronto, Toronto, ON

Department of Pharmacy, Sunnybrook Health Sciences Centre, Toronto, ON

Background: Some Canadian institutions have interpreted NAPRA and USP 797 guidance as requiring unique stability data for each manufacturer's brand to determine the beyond-use-date for sterile products. However, no studies have been done to validate the effect of manufacturer variations on cefazolin stability.

Objective: To evaluate if variations in drug manufacturer contributes to variations in cefazolin admixture stability.

Methods: We conducted a systematic review and meta-analysis of studies assessing the stability of cefazolin sodium in sterile preparations. We searched the PubMed, Scopus, and EMBASE for all studies (in English) from January 1950 to October 2017 and extracted data pertaining to 
the lab performing the study, study days, percent of drug remaining, drug manufacturer, temperature of storage (refrigerated or room temperature), container (polyvinylchloride minibags, glass, or polypropylene syringe), diluent (dextrose 5\%, normal saline or sterile water) and drug concentration. Eligibility criteria included: stability quantified using high pressure liquid chromatography, absence of freezing and thawing, single drug admixtures, and full text availability. We performed multiple linear regression on the percent remaining reported in cefazolin stability studies.

Results: Our search revealed 3449 studies. Duplicates were removed, and a total of 8 studies met our inclusion criteria resulting in 172 data points. Six different manufacturers were recorded for cefazolin (Eli Lilly, Smith Kline, Bristol-Myers, Apothecon, Novopharm, Apotex). Multiple linear regression demonstrated that manufacturers, container, diluent and concentration do not significantly impact cefazolin stability, while study day, temperature and lab did significantly impact stability (Table).

Conclusions: Our evidence suggests that manufacturer differences do not significantly contribute to variations in stability. Future research is needed to investigate the role of other contributing factors identified.

For the table that goes with this abstract, please see Abstract Appendix, available at https://www.cjhp-online.ca/index.php/cjhp/issue/view/125/ showToc

\section{Opioid Selection in the Neonatal Intensive Care Unit: Morphine versus Fentanyl: Impact on Total Opioid Exposure and Time to Enteral Feeds}

Brunton $J^{1}$, Gerges $S^{2}$, Dunn $M^{2}$, Banihani $R^{2}$, Choudhury ${ }^{2}$ ${ }^{1}$ Sunnybrook Health Sciences Centre, Pharmacy Department, Toronto, ON ${ }^{2}$ Sunnybrook Health Sciences Centre, Women and Babies Program, Toronto, ON

Background: Opioids are often used to treat neonatal pain. However, there is limited information to guide initial opioid selection in the neonatal intensive care unit (NICU).

Objective: The objective of this study is to determine if there is an association between initial opioid selection and time to full enteral feeds or total opioid exposure.

Methods: A retrospective chart review was conducted of neonates who were admitted to a level 3 academic perinatal critical care unit and received morphine or fentanyl continuous infusions between October 1, 2015 and April 1, 2017. Primary outcomes were time to enteral feed volume of 120 $\mathrm{mg} / \mathrm{kg} /$ day and total duration of opioid exposure. Secondary outcomes included cumulative opioid dose. Cumulative doses were compared between groups using a conversion factor of 1:60 to convert fentanyl to morphine equivalents. Outcomes were compared between neonates who received morphine and those that received fentanyl using appropriate statistical tests (t-test, Wilcoxon-Mann-Whitney test or chi-square test).

Results: There were no significant differences in primary outcomes between groups. The median time to enteral feed volume of $120 \mathrm{~mL} / \mathrm{kg} /$ day was 13 days in the morphine group $(\mathrm{n}=19)$ and 15 days in the fentanyl group $(n=19)$. The median duration of opioid exposure was 11 days in the morphine group and seven days in the fentanyl group. Neonates in the fentanyl group received significantly more cumulative opioid (median: $6015 \mathrm{mcg} / \mathrm{kg}$ morphine equivalents) compared to neonates in the morphine group (median: $1646 \mathrm{mcg} / \mathrm{kg})(\mathrm{p}<0.0001)$.

Conclusions: Initial opioid selection was not associated with a difference in primary outcomes of time to achieve feed volume of $120 \mathrm{~mL} / \mathrm{kg} /$ day or duration of opioid exposure. Neonates in the fentanyl group received significantly larger cumulative opioid doses compared to morphine. The clinical significance of larger cumulative opioid doses is currently unclear. Future neurobehavioural outcome studies will be performed in this patient population.

\section{The Prevalence of Mortality due to Rebound Toxicity after "Treat and Release" Practices In Prehospital Opiate Overdose Care: A Systematic Review}

\author{
Greene $J A^{1,2}$, Deveau $B J^{1}$, Dol JS $S^{1}$, Butler $M^{1}$
}

${ }^{1}$ Dalhousie University, Halifax, NS

${ }^{2}$ Emergency Health Services, Halifax, NS

Background: Traditionally, patients who have overdosed on opiated that are managed by emergency medical services (EMS) are treated with naloxone, provided ventilatory support and transported to hospital. However, some EMS agencies have allowed paramedics to not transport if patients have the capacity to refuse transport.

Objectives: The safety of this practice has not been examined by a systematic review. Therefore, we aimed to determine the prevalence of mortality and serious adverse events within 48 hours of EMS treat and release due to suspected rebound opiate toxicity after naloxone administration.

Methods: A systematic search was performed on May 11th 2017 in PubMed, Cochrane Central, Embase and CIHAL using search strategies developed with the aide of a health sciences librarian. No search limits were applied. Included studies were hand searched. Two authors conducted the screening, selection and data extraction process. Discrepancies were resolved via discussion. A modified QUIPs tool was used to evaluate risk of bias. Analysis for prevalence of outcomes were preformed.

Results: 1401 records were screened after duplicate removal. Eighteen full text studies were reviewed with 8 selected for inclusion. Included studies had a low risk of bias. The prevalence of mortality within 48 hours was so infrequent that it could not be quantitatively meta-analyzed. There were 4/4912 (0.00081\%) total reported deaths of suspected rebound etiology from included patients across all studies. Only one study reported on adverse events of patients released on scene. This study found no incidence of adverse events from their sample of 71 released patients.

Conclusion: The prevalence rate of mortality or serious adverse events due to suspected rebound toxicity was very low. Therefore, we conclude this practice may be considered as an alternative to traditional transport. Additional prospective studies need to be performed to strengthen knowledge around adverse events.

\section{A Pan-Canadian Study on the Compounded Medicines Most in Need of Commercialized Oral Pediatric Formulations}

Autmizguine ${ }^{1,2,3}$, Allakhverdi $Z^{1,2}$, Gilpin $G^{1}$, Tessier J-E $E^{1}$, Giroux $D^{1}$, Lebel $D^{1,4}$, Litalien $C^{1,2,3}$

${ }^{1}$ The Rosalind \& Morris Goodman Family Pediatric Formulations Centre of the CHU Ste-Justine (The Goodman Centre), Montréal, QC

${ }^{2}$ Department of Pediatrics, CHU Ste-Justine, University of Montreal, Montréal, QC

${ }^{3}$ Department of Pharmacology, University of Montreal, Montréal, QC ${ }^{4}$ Department of Pharmacy, CHU Ste-Justine, Montréal, QC

Background: A large number of drugs administered to children have no commercially available formulations. As a result, health care providers and parents manipulate dosage forms designed for adults. Although compounding is essential to increase access to medicines for children, it can result in adverse events or therapeutic failure. There is an urgent need to undertake a mapping of the needs for child-friendly medicines in Canada. 
Objectives: To determine: 1) the most frequently compounded medicines in Canadian pediatric hospitals; 2) the challenges associated with drug compounding; and 3) medicines most in need of commercialized oral pediatric formulations.

Methods: Sixteen Canadian pediatric academic hospitals were contacted to participate in a telephone survey including 12 open-, close-ended or Likert-scale questions.

Results: Thirteen centers participated in the survey (81.3\%). Fifty-three drugs were identified as most in need of a commercialized oral pediatric formulation. Of those, 12 were reported by $\geq 4$ hospitals as a priority (Table). The most frequently reported compounding challenges were: lack of standardization, bad taste, lack of awareness of prescribers, stability of the formulation, and availability of compounding pharmacies.

Conclusions: This study highlights which drugs are most in need for pediatric oral formulations in Canada. For compounded medicines with pediatric formulations available in other countries, we are currently assessing their adequacy and partnering with pharmaceutical industry to bring them to the Canadian market. As for those medicines without pediatric formulations in Canada or abroad, we are looking for pharmaceutical partners interested in developing such formulations. Furthermore, harmonized regulations and data-sharing should be pursued to facilitate access to child-friendly medicines for the largest number of children across borders.

\section{A Comparison of Intravenous Iron Dosing Regimens for Anemia Management in Patients Undergoing Hemodialysis}

Di Vecchia $S^{l}$, Cahill F, Soong $D^{l}$

${ }^{1}$ Windsor Regional Hospital, Windsor, ON

${ }^{2}$ Cornwall Community Hospital, Cornwall, ON

Background: Anemia is common in patients undergoing hemodialysis and can result in decreased quality of life, cardiovascular events, and need for blood transfusions. Guidelines recommend the use of intravenous (IV) iron and erythropoietin stimulating agents (ESAs) for anemia management. Optimization of doses used for IV iron and ESAs is warranted, as both are associated with adverse reactions and significant costs.

Objective: The objective of this study was to compare the efficacy of a lower once weekly dose $(62.5 \mathrm{mg})$ of IV sodium ferric gluconate versus the standard dosing regimen (125mg of IV sodium ferric gluconate) utilized at our institution.

Methods: A retrospective, observational, cohort study was conducted on patients receiving hemodialysis. Primary outcome was hemoglobin level (g/L) at 3 months. Secondary outcomes included mean weekly ESA use (units), need for blood transfusions (\%), and mean cumulative iron dose (mg) over the 3-month intervention period.

Results: Of the 102 eligible patients, 52 patients received the once weekly low-dose regimen and 50 patients received the once weekly standard-dose regimen. Multiple linear regression analysis demonstrated that IV iron-dosing regimen was not a significant predictor of hemoglobin level at 3 months $(\mathrm{p}=0.859)$. Mean hemoglobin levels $(\mathrm{g} / \mathrm{L})$ in the once weekly low-dose and once weekly standard-dose regimens were $112.58 \pm 12.63$ and $114.68 \pm 10.32$, respectively. Mean weekly ESA use (units) was significantly higher in the once weekly low-dose regimen (12 686.81 \pm 6774.07 vs. $7040 \pm 7392.66 ; \mathrm{p}<0.001$ ). Number of patients requiring transfusions did not differ significantly between the two dosing regimens ( $11.32 \%$ vs. $20 \%$; $\mathrm{p}=0.367$ ).
Conclusion: Neither of the two IV iron-dosing regimens was associated with lower hemoglobin levels. Analysis suggests this may be due to the higher ESA use in the once weekly low-dose regimen. Based on these results, it may be more optimal to continue with the standard once weekly dosing regimen.

\section{User Satisfaction Regarding Standard Assessment Tool for Field-Based Pharmacy Training}

Halapy $H^{1,4}$, Lee $A^{1}$, Manson $K^{3}$, Spizzirri $D^{2}$, Tolmie $A^{3}$

${ }^{1}$ Leslie Dan Faculty of Pharmacy, University of Toronto, Toronto, ON

${ }^{2}$ Ontario College of Pharmacists, Toronto, ON

${ }^{3}$ School of Pharmacy, University of Waterloo, Waterloo, ON

${ }^{4}$ Hospital Pharmacy Residency Forum of Ontario, Toronto, ON

Background: A province-wide standard competency-based assessment tool with companion glossary was collaboratively developed and implemented by schools of pharmacy, residency training sites and provincial licensing body within the last year in order to improve consistency of learner performance assessments.

Description: A user (preceptor/assessor, learner) satisfaction survey was conducted post-implementation of the province-wide assessment tool in order to assess user opinions regarding its usability and overall satisfaction.

Action: The survey questions were derived through a collaborative process including review by assessment experts and typical assessment tool users. After completion of each field-based final assessment utilizing the tool, preceptors/assessors and learners (students/residents) were invited via email to participate in the survey. A reminder email was sent at a scheduled follow-up time to encourage survey completion.

Evaluation: Preliminary survey results $(\mathrm{n}=236)$ showed the following: the layout of the tool was easy to follow (87\% preceptors/assessors, $94 \%$ learners), the tool's accompanying glossary was seen as helpful (83\% preceptors/assessors, 59\% learners), the tool accurately captures learner's performance ( $75 \%$ preceptors/assessors, $53 \%$ learners), the tool does not have redundant areas of assessment (80\% preceptors/assessors, $73 \%$ learners), and the tool is not missing important content areas of assessment (73\% preceptors/assessors, $83 \%$ learners). Seventy-two percent of preceptors/assessors were satisfied with the tool while $54 \%$ of learners were satisfied with the tool. The majority of comments indicated the tool was well designed, comprehensive and fair. A suggestion was to review the length.

Implications: Users indicated that the province-wide assessment tool is usable. The tool's measurement domains were generally seen to be appropriate. Preceptors were more satisfied overall with the tool versus learners. Work continues to determine details around these results and to further validate the province-wide assessment tool.

\section{Pharmacy Student-Led Best Possible Medication History Quality Audit}

Smith $A^{1}$, Jones $B-F^{2}$, Goodine $C^{2}$

${ }^{1}$ College of Pharmacy, Dalhousie University, Halifax, NS

${ }^{2}$ Horizon Health Network, Dr. Everett Chalmers Regional Hospital,

Fredericton, $N B$

Background: In May of 2016, our hospital implemented a change in practice and reconciliation of the Best Possible Medication History $(\mathrm{BPMH})$ resulted in physician orders. The majority of $\mathrm{BPMH}$ are collected and documented by a registered nurse $(\mathrm{RN})$ in our hospital and although the rate of BPMH documentation was good, reconciliation by 
physicians was below target. There was a perception that the quality of BPMH by an RN differed based on the unit where it was completed. A BMH quality audit was undertaken to explore this perception.

Description: Quality audits are not part of our regular pharmacy operations therefore, a second-year pharmacy student intern was hired to lead the BPMH audit under the supervision of a staff pharmacist.

Action: The pharmacy student completed site-specific BPMH training and had their skills validated by a staff pharmacist. Convenience sampling was used to identify eligible patients. The pharmacy student obtained verbal consent to conduct a second BPMH and quality was determined by comparing the two independent BPMH (nurse and pharmacy student) to identify discrepancies. Discrepancies were reviewed, classified, and reconciled by a staff pharmacist. Discrepancy rates were calculated based on number of home medications and descriptive statistics were used to report total discrepancies, and to compare results between units.

Evaluation: Twenty BPMH interviews were repeated for 2 clinical programs; 10 from each program. Ninety-five discrepancies were evaluated; 83 documented by nursing staff, and 12 by the pharmacy student. Discrepancy rate for BPMH by an RN 38.8\% in one program and $38.7 \%$ in the other.

Implications: A pharmacy student successfully conducted a BPMH quality audit. The audit demonstrated that there was an opportunity to improve BPMH by RNs and that there was no difference in BPMH quality between programs. Audit results will be shared within our facility and used to inform our interventions.

\section{A Work-Sampling Study of Clinical Pharmacists}

Wong $D^{1}$, Feere $A^{1}$, Dahri $K^{1,2}$, Partovi $N^{2}$, Yousefi $V^{2}$

${ }^{1}$ University of British Columbia, Vancouver, $B C$

${ }^{2}$ Vancouver Coastal Health, Vancouver, $B C$

Background: As Canadian pharmacists have gradually expanded their scope of practice, this has also increased the demand on pharmacists' time. There is a current lack of literature examining the tasks that clinical pharmacists perform in Canada and how much time each task typically consumes. It is unclear whether there are indirect patient care activities that may be reassigned to support staff or regulated technicians in order to allow more time for direct patient care activities.

Objective: To quantify the total amount of time that clinical pharmacists spend on direct and indirect patient care activities and determine which activities require the most time.

Methods: An observational work-sampling study was conducted at two urban hospitals. Trained observers followed clinical pharmacists individually for 1.5-4 hours in a variety of different wards and recorded their activities in 1-minute intervals and sorted each activity as a direct or indirect patient care activity. Simple descriptive statistics were used to analyze the time consumed by each activity.

Results: 2044 minutes of activity from 11 pharmacists were recorded. $82.8 \%$ of their time was spent on direct clinical activities, such as: $40.9 \%$ assessment and evaluation (includes $9.9 \%$ and $17.0 \%$ on reviewing paper charts and computer systems, respectively), 23.3\% rounds, $9.5 \%$ therapeutic interventions. Only $17.2 \%$ of their time was spent on indirect clinical activities, such as: $4.6 \%$ walking, $2.7 \%$ looking for something.

Conclusions: Although it was an excellent finding that pharmacists spend minimal time on indirect activities $(17.2 \%)$, the majority of the time they spent on direct clinical activities was assessment and evaluation - namely, review of paper charts and computer systems. An electronic medical record as well as technicians may be beneficial in organizing pertinent patient data for quicker chart reviews and workups.

\section{Development and Evaluation of an Anticoagulation Education Program for Pharmacists}

\author{
Kertland $H^{1,2}$, Dewhurst $N F^{1,2}$, Tom $E^{2}$, Halapy $H^{1,2}$, Yee $R^{l}$, Duan $P^{l}$, \\ Tsoi $V^{1}$, Babaei-Rad R', Chant $C^{1,2}$ \\ ${ }^{1}$ Leslie Dan Faculty of Pharmacy, University of Toronto, Toronto, ON \\ ${ }^{2}$ St. Michael's Hospital, Toronto, ON
}

Background: As part of a broader practice standardization project, an education program was developed. A series of therapeutic topics with focus on high risk medications were identified for practice standardization. Anticoagulants are high risk medications that require pharmacists to have minimal baseline knowledge of in order to identify and resolve related drug therapy problems (DTPs).

Description: To ensure pharmacists possess the required knowledge and skills, an anticoagulation education module was developed, implemented and evaluated.

Action: The anticoagulation education module consisted of a voiced-over slideshow presentation, which included supporting institutional policies and procedures. The education module underwent review and feedback from expert and typical pharmacist users prior to deployment. Assessment of the pharmacist's knowledge and skills consisted of a 20-question multiple choice test that was administered both at baseline and after review of the module. Point-biserial (p-bis) and p-values were used to ensure test question validity and reliability. Pharmacists were required to score at least $80 \%$ on the post-module test. Program evaluation consisted of a questionnaire asking about the pharmacist's own confidence and that of their colleagues to identify and resolve anticoagulation related DTPs, and the perceived value of the program.

Evaluation: Fifty-one pharmacists completed the pre- and post-module tests. Post-module completion, the average test score increased from $76 \%$ to $90 \%$. The majority of the pharmacists (49/51 [96\%]) passed the test. Responses from the post-module questionnaire indicated that pharmacists were overall confident in their own and colleagues' ability to identify and resolve anticoagulation DTPs, and perceived the program as beneficial to improve patient care and safety.

Implications: The results suggest that pharmacists benefitted from an anticoagulation education program. Completion of the education module and post-module test are now mandatory for all new staff. Based on the program's success, future modules on different therapeutic topics are in development.

\section{Évaluation de l'oculométrie comme outil de rétroaction en validation pharmaceutique : étude pilote}

Goulois $S^{1}$, Porteils $C^{1}$, Thibault $M^{1}$, Lebel $D^{1}$, Bussières $J F^{1,2}$

${ }^{1}$ Département de pharmacie et Unité de recherche en pratique

pharmaceutique, CHU Sainte-Justine, Montréal, QC

${ }^{2}$ Faculté de pharmacie, Université de Montréal, Montréal, QC

Contexte : L’oculométrie regroupe un ensemble de techniques permettant d'enregistrer les mouvements oculaires.

Objectif : Évaluer la concordance des données recueillies par oculométrie selon deux méthodes d'analyse de données.

Méthodologie : Étude descriptive. Une simulation de validation pharmaceutique du dossier pharmacologique avec interactions en personne et au téléphone (< 15 minutes, 12 ordonnances de médicaments) a été menée auprès de 16 participants. Les données recueillies par l'oculomètre (Redn, Sensomotoric Instruments, Teltow, Allemagne) ont été couplées par le logiciel utilisé à une captation de l'écran de validation vu par les participants et ces vidéos ont été analysées et recodées manuellement par deux assistantes de recherche afin d'identifier les champs d'intérêt 
regardés par les participants. Nous avons évalué la concordance (Kappa) de périodes de validation active de champs d'intérêt du dossier pharmacologique entre les données issues de l'analyse de la captation vidéo et les données brutes de l'oculomètre. Une valeur de $\mathrm{p}$ inférieure à 0,05 est considérée statistiquement significative.

Résultats : Seulement 13 des 16 captations vidéos ont permis de générer des données exploitables pour un total de 903 paires de périodes de validation active. La concordance est partielle entre la captation vidéo et les données brutes de l'oculomètre soit, en ordre décroissant de champs d'intérêt du dossier pharmacologique: posologie $(0,633)$, équivalence $(0,499)$, date d'ordonnance $(0,490)$, dose $(0,469)$, dosage $(0,171)$, médecin prescripteur $(0,079)$, trame $(0,066)$.

Conclusion : Il existe une faible concordance entre les données issues de l'analyse de la captation vidéo et les données brutes de l'oculomètre. Nous pensons que l'oculométrie représente un outil potentiellement très utile pour offrir une rétroaction en temps réel à un pharmacien ou un assistant-technique en pharmacie lors de la validation des ordonnances. Toutefois, il est nécessaire d'identifier une plate-forme logicielle mieux adaptée aux besoins de recherche.

\section{Qualitative Thematic Analysis of Interprofessional Perspectives on Clinical Pharmacy Key Performance Indicators}

Fernandes $O^{1,2}$, Raymond $C^{3}$, Mourao $D^{1}$, Meade $A^{4}$, Toombs $K^{5}$, Slobodan $\mathcal{Y}^{5}$, Hao B', Sreeskantharajan $S^{2}$, Poggemoeller $K^{6}$, Attfield E', Gorman $S^{8,6}$

${ }^{1}$ University Health Network, Toronto, $O N$

${ }^{2}$ Leslie Dan Faculty of Pharmacy, University of Toronto, Toronto, ON

${ }^{3}$ Manitoba Centre for Health Policy, University of Manitoba, Winnipeg, MB

${ }^{4}$ Nova Scotia Health Authority, Halifax, NS

${ }^{5}$ Alberta Health Services, Red Deer, $A B$

${ }^{6}$ University of British Columbia, Vancouver, $B C$

${ }^{7}$ Lower Mainland Pharmacy Services, Surrey, BC

${ }^{8}$ Interior Health Authorits, Kelowna, BC

Background: Eight national clinical pharmacy key performance indicators (cpKPIs) were developed by hospital pharmacists to advance pharmacy practice and improve the quality of patient care. Six of the eight cpKPIs have been adopted by the CSHP Excellence program. Interprofessional feedback is essential to optimize, refine and effectively communicate the importance and meaning of the cpKPIs.

Description: To identify, on a national scale, qualitative themes related to interprofessional perspectives on cpKPIs and specific areas for improved cpKPI description.

Action: This was a prospective, multi-centre, multi-province, qualitative descriptive study. Stakeholders were defined as a healthcare professional or administrator who interacts with a hospital pharmacist regularly and/ or is involved in the measurement of quality/performance indicators. Focus group and individual interviews were conducted to gather interprofessional stakeholder feedback about the cpKPIs with a semi-structured interview guide. All discussions were audio-recorded and transcribed. Two investigators conducted the qualitative thematic analysis using NVivo software.

Evaluation: Thematic analysis using feedback provided by 92 participants across 4 Canadian provinces revealed 8 major themes and 13 sub-themes related to interprofessional perceptions of cpKPIs (See Table 1). Study participants included a wide variety of stakeholders including physicians, nurses, allied health professionals, hospital administrators, and nonhospital pharmacists.
Implications: Thematic analysis of interprofessional cpKPI perspectives revealed valuable opportunities to refine the cpKPIs with a focus on their importance to support the need for pharmacists and their patient care role. The analysis also identified specific description enhancements to improve comprehension of the cpKPIs by an interprofessional audience. These perspectives can serve to inform implementation of cpKPIs nationally.

For the table that goes with this abstract, please see Abstract Appendix, available at https://www.cjhp-online.ca/index.php/cjhp/issue/view/125/ show $T$ oc

\section{Enabling Expanded Scope in Hospital Practice: Implementation of a Pharmacist Modification Orders Protocol}

\section{Carlin S, Wynne C, Tonkin M}

Hamilton Health Sciences, Hamilton, ON

Background: The revised Pharmacy Act and Pharmacy Act Regulations expanded pharmacists' scope of practice in Ontario to include prescribing. Pharmacists are not listed as prescribers in the Public Hospitals Act Regulations which currently restricts pharmacists from exercising their expanded scope in the hospital setting.

Description: A protocol was developed at our institution to provide a mechanism for pharmacists to modify medication orders for inpatients. Pharmacists use "pharmacist modification" heading to write an order in the patient chart that the pharmacist is authorized to write, based on their clinical judgment and scope of practice. Pharmacist modification orders may include: change in dose, dosage form, directions for use, route of administration, concentration, diluent or rate of administration through a parenteral route, administration time or medication hold. Pharmacists use "pharmacist suggests" heading to make a suggestion to the medical team when the pharmacist is not authorized to independently write an order.

Action: The Pharmacist Scope of Practice working group reviewed practice at other hospitals within and outside of Ontario. Building on current collaborations with physicians, pharmacists and nurses, the working group ensured organization readiness for the protocol through stakeholder discussions and meetings. Approval and endorsements were obtained from appropriate pharmacy, interprofessional, and medical advisory committees followed by education sessions for pharmacists and clinical teams and subsequent implementation in July 2017.

Evaluation: A one-day audit of pharmacist orders was completed approximately one month after protocol implementation. Pharmacists wrote 188 orders: 47 modifications, 102 suggestions, 22 automatic substitutions and 17 others. Interventions included: 32\% initiations, $23 \%$ dose adjustments, $16 \%$ medication management and $12 \%$ discontinuations. Pharmacist feedback has been positive with respect to patient care and workflow.

Implications: This protocol has enabled pharmacists to expand their scope of practice in order to improve effectiveness, safety, efficiency and costeffectiveness of patient care.

\section{Design, Implementation, and Evaluation of a Clinical Pharmacy Key Performance Indicator Tracker: DIE-cpKPI Study}

Slavik $R S^{1,2}$, Scott, $K^{2}$, Gorman $S K^{1,2}$, Bruchet, $N^{1,2}$, Dalen $D^{1,2}$, Hutton L3, Fernandes $O^{3,4}$

${ }^{1}$ Pharmacy Services, Interior Health, Kelowna, BC 
${ }^{2}$ Faculty of Pharmaceutical Sciences, University of British Columbia, Vancouver, $B C$

${ }^{3}$ Pharmacy Department, University Hospital Network, Toronto, ON ${ }^{4}$ Leslie Dan Faculty of Pharmacy, University of Toronto, Toronto, ON

Background: High-quality evidence shows clinical pharmacists providing Canadian consensus clinical pharmacy key performance indicator (cpKPI) activities with their inter-professional team improve health and economic outcomes. A cpKPI tracker is required to measure health service delivery, advance pharmacy practice, and improve patient care.

Objective: To design, implement, and evaluate a theory-informed and evidence-based professional behavior change intervention (BCI) used to implement a cpKPI tracker.

Methods: Prospective, quasi-experimental, one group, PRE/POST study at three hospitals from January 30th to June 30th, 2017. The primary outcome was the mean difference in scores of a 45 -item PRE and POST $\mathrm{BCI}$ questionnaire scores. Secondary outcomes included the mean difference in PRE and POST BCI questionnaire scores across each of the 14 domains of the Theoretical Domains Framework (TDF), percentage of clinical pharmacists "satisfied" with the BCI, and percentage of discharged patients receiving cpKPI interventions during 2-month pilot period.

Results: Twenty four (80\%) of representative pharmacists participated in the study. The mean (95\% confidence interval) difference in PRE and POST BCI questionnaire scores was $-0.75(-0.65$ to -0.84$)$. Scores were significantly reduced across all TDF domains except "intentions". Overall, $71 \%$ of the participants were "satisfied" with the intervention provided. Overall, 634 of $7264(8.7 \%)$ of discharged patients received at least one cpKPI intervention. Participation in interprofessional patient care rounds, implementing a PC plan, resolving DTPs, and providing inpatient education and counseling were provided most frequently. Admission and discharge medication reconciliation and discharge education and counselling were provided less frequently.

Conclusions: An evidence-based BCI was systematically designed, efficiently implemented, and successfully evaluated. The BCI reduced perceived barriers to $\mathrm{cpKPI}$ tracking, and pharmacists were satisfied with the BCI used to implement the cpKPI tracker. Pilot data captured was successfully retrieved and reported. Several hypotheses may explain the frequency of cpKPI activities reported, which will guide additional pre-implementation work.

\section{Assessing the Perception and Implementation of Continuous Quality Improvement in Pharmacy Professionals: A Pre-Safety IQ Initiative}

Boucher $A^{1,2}, H_{o} C^{1,2}$, Hardy ${ }^{3}$, Carlson $R^{3}$, Eros $R^{3}$

${ }^{1}$ Institute for Safe Medication Practices Canada, Toronto, ON

${ }^{2}$ Leslie Dan Faculty of Pharmacy, University of Toronto, Toronto, ON

${ }^{3}$ College of Pharmacists of Manitoba, Winnipeg, $M B$

Background: Safety IQ is a standardized continuous quality improvement (CQI) program in Manitoba designed to prevent medication incidents from happening in pharmacies.

Description: The objective of this project was to explore the current perceptions, benefits, barriers, and experience of CQI programs in pharmacy professionals prior to the launch of the province-wide Safety IQ initiative.

Action: We administered a 28-item online questionnaire over a two-week period to all registered pharmacists and pharmacy technicians in Manitoba. We conducted descriptive statistics and qualitative thematic analysis, accordingly, on the responses collected.
Evaluation: We collected 125 responses, with 32\% pharmacy managers, $56.80 \%$ staff pharmacists, and $11.20 \%$ pharmacy technicians. Pharmacy professionals had a fairly positive perception of CQI program and its associated benefits to patient care and safety. They viewed CQI program as a platform for communication and shared leaning with the ultimate goal of preventing medication incidents. There were concerns regarding CQI program implementation, such as the potential requirement for additional financial and human resources, as well as fear of reporting and discussing incidents. Time was considered to be the greatest challenge in CQI program implementation. Pharmacy professionals preferred a simple, efficient CQI program, and perceived that support from management would be required for its sustainability. They shared a wide range of experiences with current CQI programs in their practice.

Implications: Implementation of CQI programs vary widely in Manitoba and most seem to be generally informal and internally focused. Despite concerns in increased resource requirements, pharmacy professionals appeared to be open and supportive of a standardized CQI program. They expect such a program will provide benefits including a more positive work environment, increased public trust, and a reduction in medication errors. This project provides support for the implementation of Safety IQ as a standardized CQI program to improve patient and medication

\section{From "Dot" to "Dot Com": Navigating Pharmacist Handoff in a Digital Era}

Lui J, Patel M, Ahmed E, Fontana P, Bahadur D, Karas A, Wood C Humber River Hospital, Toronto, ON

Background: Effective communication is an important aspect of providing exceptional patient-centered care. During transition of care points, a requirement for timely, accurate and important information to the receiving provider is necessary. Referred to as a 'handoff', transfer of care occurs when two or more health care professionals exchange information pertinent to a patient's care. The shift to computerized provider order entry (CPOE) and a vision of "Lean, Green and Digital" for our new hospital provided an opportunity to evaluate and standardize our pharmacist handoff processes.

Description: Our previous pharmacist handoff processes involved various methods, dependent on the pharmacist at the initial point of care. A "sticker dot" on the original paper order was the most common (greater than 50\%) method of communication. The dot would be accompanied with handwritten notes. The aim of our intervention was to provide congruency between pharmacists at handoff; ensuring communication was clear, concise, timely, and inclusive.

Action: A group of key stakeholders including the pharmacy managers, director, pharmacists, drug utilization, and informatics pharmacists led this project. A baseline documentation survey was sent to all pharmacists to address the needs and concerns. Utilizing these results, we were able to facilitate automated reports that were customizable; allowing for prioritization of handoff interventions. Extensive educational sessions were held for pharmacists to ensure uptake of the new handoff tool.

Evaluation: The Plan-Do-Study-Act (PDSA) cycle was used to ensure the tool was relevant and useful. Follow up educational sessions were required for front line pharmacists to use the handoff tool appropriately and effectively. Our pharmacists utilize the automated tool on a daily basis and have implemented it into their daily workflow processes.

Implications: The success of standardizing the electronic handoff processes has allowed for seamless transfer of care between pharmacists. Future enhancements may focus on documentation standardization for pharmacists. 